\title{
Supraliminal but No Subliminal Priming by High-Spatial Frequency Faces in a Face-Sex Discrimination Task
}

\author{
Shah Khalid1, Ulrich Ansorge ${ }^{2}$, Matthew Finkbeiner ${ }^{3}$ \\ ${ }^{1}$ Institute of Cognitive Science, University of Osnabrück, Osnabrück, Germany \\ ${ }^{2}$ Faculty of Psychology, University of Vienna, Vienna, Austria \\ ${ }^{3} A R C$ Centre of Excellence in Cognition and its Disorders, Macquarie University, Sydney, Australia \\ Email: shah.khalid@uni-osnabrueck.de
}

Received 2 July 2015; accepted 13 September 2015; published 17 September 2015

Copyright (C) 2015 by authors and Scientific Research Publishing Inc.

This work is licensed under the Creative Commons Attribution International License (CC BY).

http://creativecommons.org/licenses/by/4.0/

(c) (i) Open Access

\begin{abstract}
It has been argued that visual subliminal processing of face-inherent sex information might depend on the retino-collicular projection. In the current study, we tested this possibility. Based on the known exclusive sensitivity of the retino-collicular projection for low spatial frequencies (LSF), we derived the following predictions. First, masked and, therefore, subliminal high-spatial frequency (HSF) face primes would not be processed, but, secondly, masked unfiltered face primes, thirdly, masked LSF face primes, and, fourthly, unmasked HSF primes should be processed and should lead to a congruence effect-that is, faster responses (better performance) in congruent conditions, with face primes and face targets of the same sex, as compared to incongruent conditions, with face primes and face targets of different sexes. These predictions were borne out in altogether three experiments. In Experiment 1, we observed that masked unfiltered primes created a congruence effect and that masked HSF primes failed to do so. In Experiment 2, we found that masked unfiltered primes and masked LSF primes both created significant congruence effects of about the same size. Finally, in Experiment 3, we demonstrated that unmasked HSF primes led to a congruence effect, whereas masked HSF primes failed to do so. Also, masking led to the subliminal presentation of the primes, as indicated by the fact that our participants were unaware of the masked but not the unmasked face primes. Together, these findings are in general agreement with an assumed origin of subliminal (or unaware) face processing along the magno-cellular projection of the human visual system.
\end{abstract}

\section{Keywords}

Face Processing, Priming, Magno-Cellular Projection, High-Spatial Frequencies, Low-Spatial Frequencies 


\section{Introduction}

Phylogenetically, vision is ancient and developed to allow organisms to successfully adjust their behavior to dynamically changing environmental constraints. Even very simple organisms show basic visual capabilities of sub-serving motor control: Nematodes, for instance, exhibit phototaxis - the movement towards light. In light of the simplicity of some organisms exhibiting basic visual capabilities, it is likely that not all these forms of vision depend on visual awareness. This is difficult to demonstrate in non-human taxa. However, in humans this is feasible. First, one has to show that human participants cannot be discriminated between visual stimuli with above-chance accuracy (Reingold \& Merikle, 1988). Second, once the invisibility of a stimulus has been firmly established, one also needs to demonstrate that the invisible stimulus is processed nonetheless. This dissociation between awareness and processing has now been demonstrated in humans in numerous studies using different procedures (e.g., Dolan et al., 1996; Tamietto \& De Gelder, 2010; Weiskrantz, 1986).

One experimental procedure that has been used to demonstrate unaware vision in healthy humans is masked priming (Ansorge, Kunde, \& Kiefer, 2014; Breitmeyer \& Ögmen, 2006; Dimberg, Thunberg, \& Elmehed, 2000). In masked priming, a priming stimulus is briefly shown and subsequently its visibility is suppressed by a visual mask. For example, a pattern can be presented as a mask, with a small interval after a brief image and at the same position as the image. In this situation, discrimination of the image can be reduced to chance-level accuracy (Morris, Öhman, \& Dolan, 1999). Despite the prime’s invisibility, however, the prime can facilitate-therefore the name "prime"-(or inhibit) the processing of a subsequent visual target. Using female and male face images as primes and targets, for example, Finkbeiner and Palermo (2009) showed that presenting a gendercongruent masked prime before the visible target face facilitated the classification of the target face as male or female. This facilitation was observed relative to an incongruent condition with masked prime and target of different genders: Participants were faster to classify, for example, a female face target as female after a masked female face prime was faster to classify than after a masked male face prime. This congruence effect was found although the same participants were incapable to be successfully discriminated between the face primes. This congruence effect was also independent of attention. Although primes and targets were shown at different locations and a cue was shown to direct attention either to the prime or to the target, the position of the cue had no effect on the size of the priming effect when face stimuli were used (Finkbeiner \& Palermo, 2009). This independence of masked priming from attention would be in line with classical conceptions of automatic processing as being both aware- ness-independent and attention-independent (Posner \& Snyder, 1975; Mulckhuyse \& Theeuwes, 2010).

Importantly, unraveling the principles of unaware vision also has the potential to shed a light on questions concerning the differences between aware and unaware vision and, thus, may be of help for understanding the role of awareness in vision and the division of labor between aware and unaware processes (cf. Dehaene \& Naccache, 2001; Dixon, 1971; Greenwald, Draine, \& Abrams, 1996; Lamme \& Roelfsema, 2000). One recently highlighted characteristic of unaware vision is its sometimes seemingly stronger dependence on the presence of low spatial frequencies (LSF) than high-spatial frequencies (HSF) within the visual image (De Gardelle \& Kouider, 2010; Khalid, Finkbeiner, König, \& Ansorge, 2013). De Gardelle and Kouider (2010) presented LSF or HSF filtered images of famous and non-famous faces as primes and targets. These authors used masked and less masked/visible primes and observed that the priming effect of HSF images but not that of the LSF images depended on prime visibility where prime visibility was manipulated by using a small prime-mask interval (of $43 \mathrm{~ms}$ ) for the unaware conditions and a longer interval (of $300 \mathrm{~ms}$ ) for the aware priming conditions. More recently, Khalid et al. (2013) used masked primes in the paradigm of Finkbeiner and Palermo (2009), and found congruence effects with LSF but not with HSF primes partly confirming the data pattern found by De Gardelle and Kouider.

Regarding an explanation of the frequency-dependence of the awareness-independent masked priming effect, the fact that LSF but not HSF content which is processed points to the superior colliculi (SC) and the magnocellular visual pathway as one major origin of unaware vision because this pathway is exclusively sensitive to the LSF band (Bullier, 2001; Croner \& Kaplan, 1995; Livingstone \& Hubel, 1988). The magno-cellular projection ascends from the retinal ganglion cells. It is one of two major projections-the other one is the parvo-cellular projection - that remain largely segregated in the lateral geniculate nucleus (LGN) of the thalamus and up to cortical levels. The magno-cellular projection also branches into the superior colliculi (SC), an area of the midbrain, and from there connects to other cortical areas, such as the posterior parietal cortex, via the pulvinar of the thalamus. 
Crucially, the SC and the magno-cellular projections are comparatively more sensitive to the LSF band. This is because its retinal ganglion cells show a high degree of convergence- that is, many peripheral retinal photoreceptors converge on a single ganglion cell feeding into the magno-cellular projection (Merigan \& Maunsell, 1993). Because retinal convergence undermines the spatial resolution of the retinotopic image, the magno-cellular projection works like an LSF filter. This is different for the parvo-cellular projection which is relatively more sensitive to the HSF band, too. Also important, the magno-cellular projection with its branching via the SC has been held responsible for forms of subliminal vision (Jolij \& Lamme, 2005; Mulckhuyse \& Theeuwes, 2010; Tamietto \& De Gelder, 2010; Weiskrantz, Warrington, Sanders, \& Marshall, 1974). Take blindsight for an instance, in blindsight processing of visual information presented to the visual field regions affected by scotomae after lesions of striate visual cortex has been attributed to the subcortical branch of the magno-cellular pathway via the SC (Weiskrantz et al.). Even if the assumption that all forms of subliminal processing depend on the subcortical visual pathway via the SC is too general, as some forms of subliminal processing occur via the parvo-cellular projection (e.g., Tapia \& Breitmeyer, 2011) and in cortical regions suggested (Kouider, Eger, Dolan, \& Henson, 2009), the assumption of a collicular origin of subliminal processing might at least hold true for certain forms of face processing, such as the processing of a face's sex (Khalid et al., 2013) and its emotional expressions (Dolan et al., 1996; Tamietto \& De Gelder, 2010).

\section{Overview of the Present Study}

In the current study, we used a face-sex discrimination task (Finkbeiner \& Palermo, 2009; Khalid et al., 2013) and tested for a potential qualitative difference between supra- and subliminal face priming. If it is true that subliminal processing of masked face sexes depends on the SC but that supraliminal processing of unmasked face sexes does not depend only on the SC, we expect to see a difference between subliminal LSF and HSF face primes but not between subliminal LSF and supraliminal HSF face primes. The sex of the subliminal LSF face primes should be processed via the SC. Because this pathway is selectively sensitive to the LSF content, we expect to see a congruence effect of masked LSF faces but not of masked HSF faces. In contrast, the sex of a supraliminal face prime should be processed via the magno- and the parvo-cellular projections. Accordingly, we expect to see a congruence effect also by unmasked HSF primes.

Critically, in both masked and unmasked priming conditions, the prime-target intervals in the current study were kept constant. This is different from De Gardelle and Kouider (2010) and resolves one important question. Because masked priming effects also depend on prime-target intervals alone (Vath \& Schmidt, 2007; Vorberg, Mattler, Heinecke, Schmidt, \& Schwarzbach, 2003), it is not clear whether the different priming effects of HSFfiltered and unfiltered faces images that were found by De Gardelle and Kouider depend on the prime-target interval itself or on the visibility that increased with the interval.

Also important, we either cued the prime or the target. This was done because Finkbeiner and Palermo speculated that cueing of attention towards the prime could be critical for the priming effect of masked HSF images but not for the effect of masked LSF primes. As explained, these authors found that masked face priming did not depend on attention. However, masked object priming in an object classification task depended on attention and was selectively enhanced under prime-cued conditions (see Figure 3 of Finkbeiner \& Palermo, 2009; see also Kiefer \& Brendel, 2006; Naccache, Blandin, \& Dehaene, 2002). To explain the different influences of attention on masked-face priming during sex categorization on the one hand, and masked object and word priming in other situations on the other hand, these authors argued that only the processing of subliminal HSF content might depend on attention and that HSF content was simply not involved in the subliminal face-sex congruence effect. Possibly, directing attention could thus gate access of even subliminal HSF primes to the parvo-cellular pathway, with the consequence of allowing a congruence effect of masked HSF primes at least under cued conditions (but see Khalid et al., 2013). As it was thus theoretically possible that attention played a mediating role in the masked HSF priming effect, an attention manipulation was therefore included in the present design. In summary, the present study extends the previous findings by testing one important qualitative difference between aware and unaware priming: whether the processing of visual HSF information depends on visibility or at least on attention and whether the processing of LSF information does neither depend on visibility nor on attention.

\section{Experiment 1}

In Experiment 1, we used HSF face primes and unfiltered (broad-spectrum) face primes. All primes were 
masked and presented peripherally prior to clearly visible target faces. In a target sex discrimination task, we tested whether the masked face primes produced a congruence effect, with faster responses to congruent than incongruent prime-target pairs. This experiment basically replicates prior conditions of Khalid et al. (2013). However, in that study, the foreground-background luminance and contrast of the filtered face primes was not manipulated, although HSF filtering diminishes the luminance and contrast of a stimulus and participants are slower to process low- than high-contrast and-luminance stimuli (cf. Purushothaman, Patel, Bedell, \& Ogmen, 1998). Because lower contrast or luminance of HSF than broad-spectrum primes could thus have also diminished the efficient prime-target onset asynchrony and the priming effect in the HSF priming conditions (cf. Vorberg et al., 2003) of Khalid et al., between groups in the present Experiment 1, we manipulated the primetarget contrast and luminance of the HSF primes.

For group 1 of our participants, the HSF face primes were simply high-pass filtered versions of the unfiltered face primes. For group 2, unfiltered and high-pass filtered face primes were additionally equated for their respective overall luminance and prime-background contrast. The HSF primes were luminance and contrastequated to compensate for the potentially slower processing of a low-luminance or low-contrast as compared to a high-luminance or high-contrast stimulus. If the SC is responsible for the masked faces' congruence effect, we expected to only see the congruence effect in the unfiltered conditions but not in the HSF face priming conditions. However, if lower foreground-background contrast or luminance of the HSF face primes than the unfiltered faces primes falsely suggested the absence of the congruence effect in the HSF priming conditions of Khalid et al. (2013), we expected to find no congruence effect only in the non-adjusted conditions of group 1 but to find a congruence effect in the luminance and contrast adjusted conditions of group 2.

Also, we included visible HSF face targets in $10 \%$ of the trials for group 2 so that we could assess participants' ability to categorize these stimuli when presented consciously. The use of 10\% HSF targets had the additional advantage that the participants were forced to include HSF content in their top-down control settings. This could be an important factor because past studies of subliminal priming have shown that a top-down control setting to process the information that is contained in the primes could be critical for the priming effect (Ansorge, Horstmann, \& Worschech, 2010; Ansorge \& Neumann, 2005). For example, Ansorge and Neumann found a masked priming effect of black primes when their participants were searching for and responding to black targets but they failed to find this priming effect when their participants were searching for and responding to red targets. The use of $10 \%$ HSF targets in the current group 2 should thus ensure that the participants had to search for and process HSF content in the face stimuli and could not just simply ignore HSF information altogether.

For both groups, all primes were presented slightly shifted into the visual periphery and the targets were shown at a fixed position below the prime (cf. Finkbeiner \& Palermo, 2009; Khalid et al., 2013). In this way, we ensured that the participants could optimally focus on the targets and that attention to the targets versus to the primes could be manipulated nonetheless (by presenting the cue either at prime or target position) (see Figure 1). With respect to the latter, it is known that an abrupt-onset cue close to the threshold of detection reliably capture attention in a stimulus-driven way (Fuchs, Theeuwes, \& Ansorge, 2013; Schöberl, Fuchs, Theeuwes, \& Ansorge, 2015). The fact that these cues can capture attention to the prime's position has also been shown by Finkbeiner and Palermo (2009). These authors observed a masked priming effect of object primes on object classification if a cue captured the participants' attention towards the prime position but not if the cue was presented at (and captured attention towards) the target position. If Finkbeiner and Palermo (2009) were right and subliminal HSF content can be gated through the parvo-cellular projection if cued, we might observe a subliminal congruence effect of the HSF primes in the prime-cued conditions but not in the target-cued conditions.

\subsection{Method}

\subsubsection{Participants}

Fifty students (26 female) with a mean age of 24.6 years participated. Here and in the following experiments, all participants had normal or corrected to normal vision. Also, informed consent was obtained from the participants in all experiments, and the participants were treated in accordance with the rules of the declaration of Helsinki and APA standards. They received course credit for participation.

\subsubsection{Apparatus}

Visual stimuli were presented on a 15-inch, color flat screen display. Its refresh rate was $59.1 \mathrm{~Hz}$ with NVIDIA 


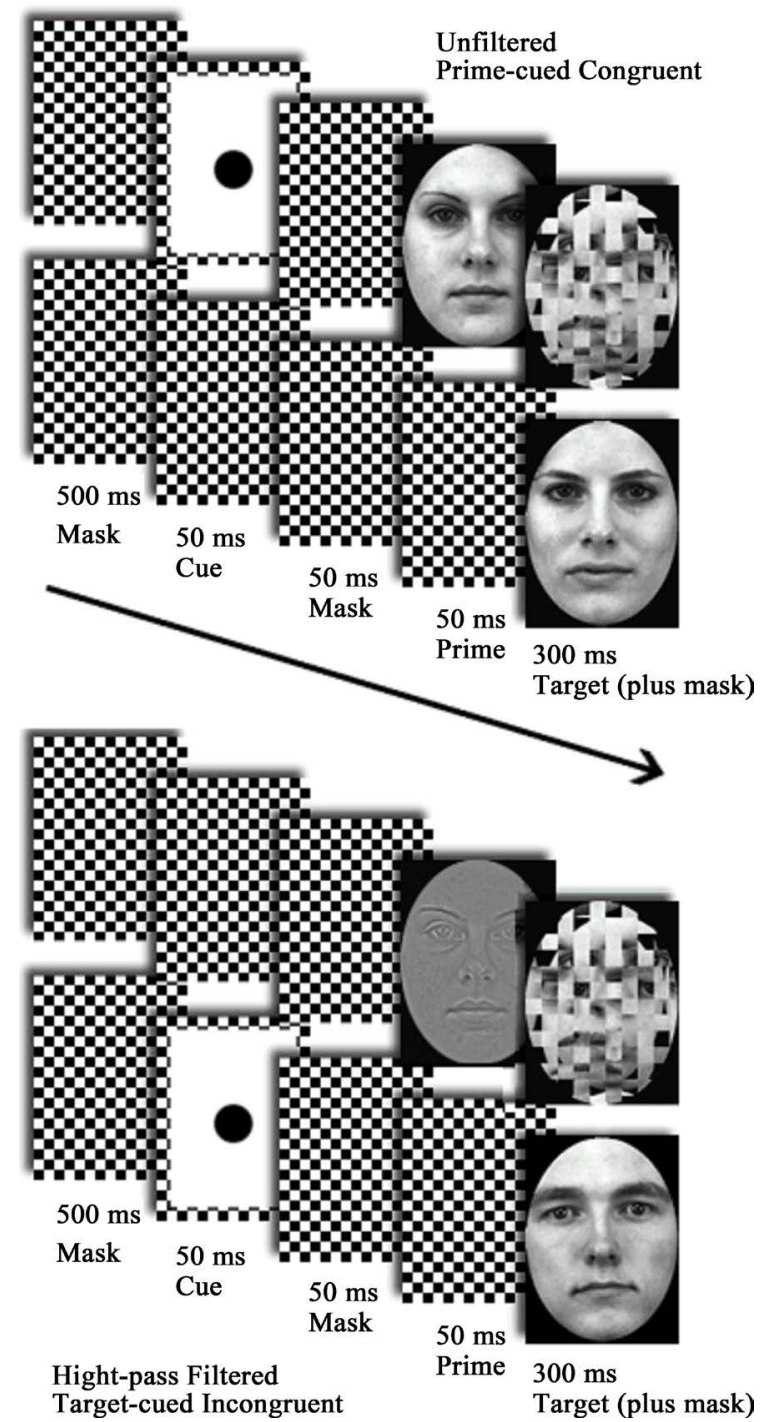

Figure 1. The upper panel shows a sequence of stimuli with an unfiltered face prime in a prime-cued congruent trial of Experiment 1 (The same conditions were used in Experiments 2 and 3). In the lower panel, a sequence of stimuli with a high-pass filtered (HSF) face prime in a target-cued incongruent trial of Experiment 1's group 1 is depicted. Primes were always presented above targets. Arrows depict the flow of time.

GeForce GT 220 (with 1024 MB) graphics adapter. The pictures that we presented had dimensions of 75 width $\times 216$ height pixels and sizes of 17.0 KB (backward mask + target). However, to avoid any buffer overload problems, all stimuli were loaded offline only once and pointers to them were used in the experimental trials. Matlab with Psychophysics toolbox -3 was used, and care was taken for correct stimulus presentation durations. Accurate timing of the displays was verified through measurement with a cathode ray oscilloscope by repeatedly presenting the stimuli for their original (experimental) duration one by one and recording their presentation duration through the oscilloscope. In all cases, the precision of presentation duration was better than one millisecond.

The participants sat at a distance of $57 \mathrm{~cm}$ from the screen in a quiet, dimly lit room, with their head resting in a chin rest to ensure a constant viewing distance and a straight-ahead gaze direction. Reaction times (RTs) were registered via the standard serial computer keyboard, placed directly in front of the participants. Target responses were registered through the keys ' $A$ ' and ' $L$ ' (covered and labeled as 'left' and 'right', respectively). The participants put their left and right index fingers on the correspondingly labeled keys. After reading the instructions and listening to the instructions explained by the experimenter, the participants pressed the spacebar with 
one of their thumbs to start the experiment, and after each trial to continue with the experiment. This provided the participants the opportunity to take breaks at their convenience by simply not pressing the space bar.

\subsubsection{Stimuli}

We used the masks, cues, unfiltered face primes, and the target face stimuli of Finkbeiner and Palermo (2009). The face targets and the unfiltered face primes wore neutral emotional expressions and were originally taken from the Karolinska Directed Emotional Faces (KDEF) database (Lundqvist, Flykt, \& Öhman, 1998), and equated to one another for foreground-background luminance and contrast (see Figure 2). Additionally HSF images of high-pass filtered face primes were used. The HSF primes were created from the unfiltered face primes using ImLab, an open source graphical application for scientific image processing (ImLab 2.3.3, MIT License, 2009). The HSF primes were identical to the unfiltered face primes in resolution and size (see Figure 2).

During high-pass filtering, first the face areas from the unfiltered face prime images were extracted from their oval boundaries through cropping. Next, all low spatial frequencies $(<3$ cycles/image) were eliminated by applying a Laplacian of Gaussian function with a standard deviation of 1 (for the resulting band-widths, see Figure 3). The lower cut-off frequency was based on neurophysiological findings. The collicular responses to Gabor gratings tail off at about 3 cycles/degree (e.g., Tailby et al., 2012), closely corresponding to our cut-off. The resultant filtered face images were then re-cropped behind the oval layer. These HSF primes had a lower luminance and a lower foreground-background contrast than the unfiltered primes (see Figure 2). To control for these confounding influences of luminance and contrast, we tested only half of the participants (group 1) with the uncorrected HSF primes, and the other half of the participants (group 2) with corrected HSF primes, which we equated with the unfiltered face primes for luminance and foreground-background contrast, through adjusting the input gray level value of the uncorrected HSF primes to the mean gray level value of the unfiltered primes. As a consequence, the distribution and mean gray level of pixels of corrected HSF primes was shifted towards white, resulting in brighter HSF images (see Figure 2, panel b). This should compensate for the known delay of

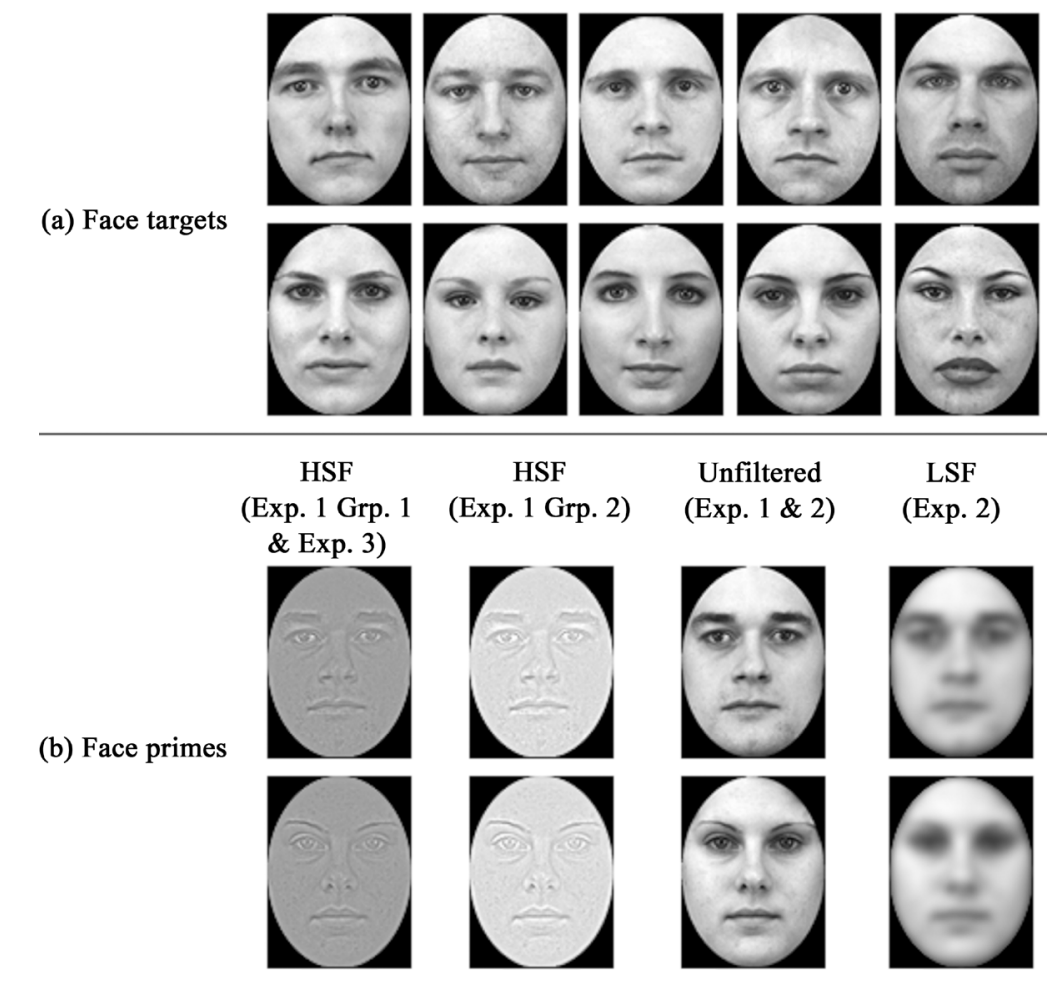

Figure 2. Panel (a): male and female face targets. Panel (b): face primes; from left to right: high-pass filtered (HSF) face primes (used in Experiment 1's group 1 and in Experiment 3), luminance and foreground-background contrast equated high-pass filtered (HSF) face primes (used in Experiment 1's group 2), unfiltered face primes (used in Experiments 1 and 2), and low-pass filtered face primes (used in Experiment 2). 


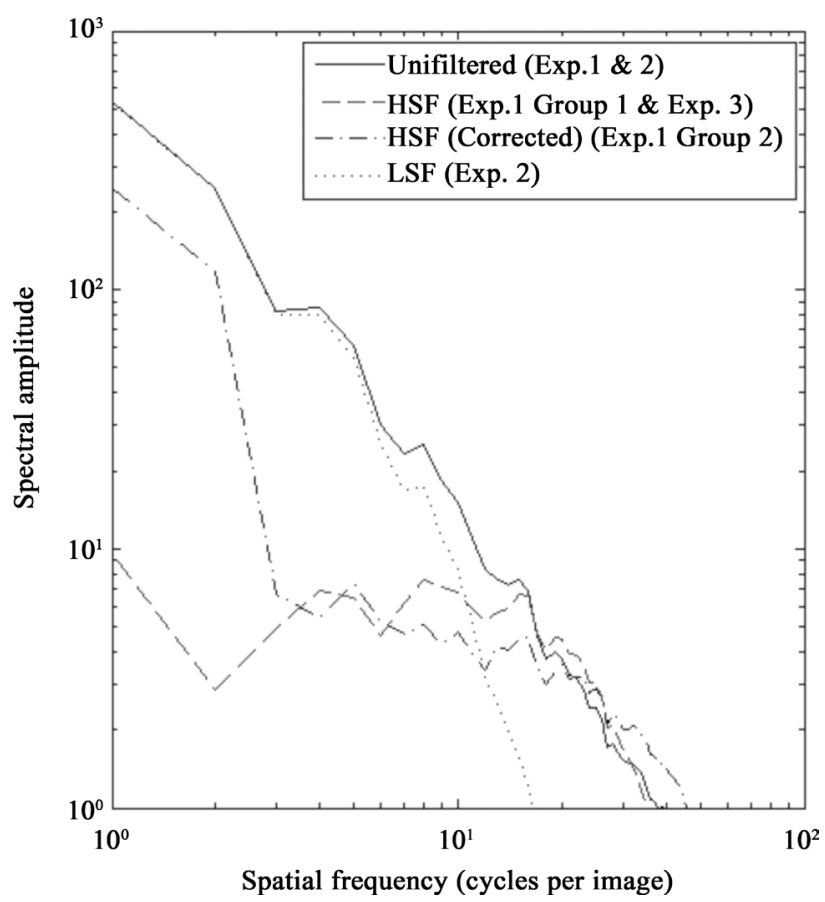

Figure 3. Log-log plots of radially averaged Fourier spectral amplitude on the y axis as a function of spatial frequency (in cycles per image) on the x axis (cf. Graham \& Redies, 2010; Olshausen \& Field, 1996) for unfiltered primes of Exp.1 and 2 (solid line), HSF primes of Exp. 1 Group 1 (broken line), HSF primes of Exp. 1 Group 2 (broken and dotted line), and LSF primes of Exp. 2 (dotted line). Each line represents an average of the plots for male and female face images. As can be seen the spectral power of the HSF primes in Exp. 1 Group 2 was linearly increased, but also in the LSF part of the spectrum.

processing a low-contrast as compared to a high-contrast stimulus (cf. Purushothaman et al., 1998). In group 2, we also included $10 \%$ filler trials (analyzed separately) in which the HSF primes were used as targets. These filler trials were randomly intermixed with the unfiltered face target trials.

All of the face stimuli were cropped in an oval layer so that only the face features were presented. Each image subtended a visual angle of $3.0^{\circ}$ vertically and $2.5^{\circ}$ horizontally. The forward mask was a checkerboard pattern. The backward mask was a scrambled composite of the two unfiltered face primes.

\subsubsection{Procedure}

See Figure 1 for examples of a sequence of events in a trial. Stimuli were presented on a black screen (luminance $<0.1 \mathrm{~cd} / \mathrm{m}^{2}$ ). In each trial, two streams of stimuli were presented, one stream directly above the screen center and the other below it. The target was always presented in the lower stream and the prime always in the upper stream. The distance between the centers of the two streams was $3.2^{\circ}$.

Each trial began with a checkerboard mask for $500 \mathrm{~ms}$. Next, an unpredictable cue was shown at either prime or target location, together with the checkerboard mask at the non-cued location, for $50 \mathrm{~ms}$. The cue was used to capture attention towards the prime (in the prime-cued condition) or to the target (in the target-cued condition) (see Fuchs et al., 2013; Finkbeiner \& Palermo, 2009). After this, the checkerboard mask was shown again at both positions for $50 \mathrm{~ms}$, followed by the prime face at the upper position together with the checkerboard mask at the target's position for $50 \mathrm{~ms}$. The two face primes, one male and one female, were taken from different individuals than the face targets to avoid repetition priming (Forster \& Davis, 1984; Norris \& Kinoshita, 2008). In the final frame, the target face was presented in the lower location for $300 \mathrm{~ms}$ together with a scrambled-face backward mask in the upper (prime) location.

Across trials, prime and target sexes varied orthogonally to create congruent and incongruent conditions. In the congruent conditions, the prime face sex was the same as the target face sex. Either a male face prime was presented prior to a male face target, or a female face prime was presented prior to a female face target. In the incongruent conditions, the prime face sex was different from the target face sex. Either a male face prime was presented prior to a female face target, or a female face prime was shown prior to a male face target. 
In the target discrimination task, participants had to discriminate the sex of the target faces by pressing one of the assigned buttons (counter balanced across participants). The target discrimination task consisted of two blocks, one with HSF primes and the other one with unfiltered primes. The sequence of the two target-discrimination blocks was also counter balanced across the participants. Each of the two target discrimination blocks consisted of 20 repetitions of each combination of cueing (prime-cued, target-cued), target type (male, female), and prime-target congruence level (congruent, incongruent), for a total of 160 trials. For group 2, an additional 16 filler trial with HSF face targets was interleaved. At the end of the experiment, participants performed one block of trials in which they had to categorize the masked primes. For this prime-discrimination task, participants first categorized the visible target (just as they had in the experiment proper) and then they categorized the masked prime. The same sex-to-response-button mapping was used for the prime faces as for the target faces. The prime-discrimination task consisted of another 200 trials, 100 trials with unfiltered face primes, and the other 100 trials with HSF face primes.

Within each block, the different conditions were realized in pseudo-random sequence, with the two constraints that no particular face target was repeated in immediately succeeding trials and that no more than four trials in a row required the same target response. The experiment was run in a single session, with five short breaks, one in the middle of each block plus one after each of the first 2 blocks. The whole experiment took approximately 1 hour.

\subsection{Results}

\subsubsection{Target-Discrimination Task}

For our first analysis, only correct target responses were considered. (The filler trials of group 2, and the erroneous trials of both groups were analyzed separately, see below.) The mean RTs are depicted in Figure 4 and also detailed in Table 1. As can be seen in Figure 4, we found a congruence effect with the unfiltered primes (circular symbols) in both target-cued (filled symbols) and prime-cued (unfilled symbols) conditions of both groups, but not with the HSF primes (triangular symbols).

This was confirmed by formal analysis. Mean RTs for each participant and condition were calculated, and trials that were faster or slower than 2.5 standard deviations of the mean were discarded (3.5\%). An omnibus mixed-model ANOVA was run with the repeated-measures variables prime type (unfiltered prime vs. HSF prime), cue type (target-cued vs. prime-cued), prime-target congruence (congruent vs. incongruent), and the between-participants group variable (Group 1: uncorrected HSF primes vs. Group 2: luminance and contrast equated HSF primes). Bonferroni adjustments for multiple comparisons and the alpha level of .05 for all statistics were applied here and throughout the study.

The main effect of congruence was significant, $F(1,49)=10.45, p<0.01$, partial $\eta^{2}=0.18$. It reflected faster responses in the congruent condition $(M=494 \mathrm{~ms})$ than in the incongruent condition ( $M=498 \mathrm{~ms})$. Importantly, a significant two-way interaction of the variables prime type and congruence was observed, $F(1,49)=16.25, p$ $<0.001$, partial $\eta^{2}=0.25$. As expected on the basis of the hypothesis that subliminal priming depends on the colliculi, the congruence effect (incongruent RT minus congruent RT) was significantly larger with unfiltered primes (congruence effect $=9 \mathrm{~ms}$ ) than with HSF primes (congruence effect $=-2 \mathrm{~ms}$ ) (see also below for follow-up analyses).

We also found a significant main effect of the variable cue type, $F(1,49)=20.88, p<0.001$, partial $\eta^{2}=0.30$, as in Finkbeiner and Palermo (2009). Responses were faster in the target-cued condition ( $M=491 \mathrm{~ms})$ than in the prime-cued condition $(M=500 \mathrm{~ms})$.

Unexpectedly, we also obtained a significant two-way interaction of the variables congruence and group, $F$ (1, 48) $=7.28, p<0.01$, partial $\eta^{2}=0.13$. This interaction reflected that the overall congruence effect was only $1 \mathrm{~ms}$ in Group 1 but it was $6 \mathrm{~ms}$ in Group 2. This interaction likely reflected differences between the groups' individuals in their vulnerability to priming because numerically weaker priming effects in Group 1 were found with both filtered as well as unfiltered primes although only the filtered primes (and the inclusion of HSF filler targets) were different between these groups. No other significant effect or interaction was found, all $F \mathrm{~s}<1.00$.

To understand the two-way interaction of prime type and congruence, separate follow-up ANOVAs were run for the unfiltered face primes and for the HSF primes, respectively, each with the variables cue type, congruence, and group. For the unfiltered primes, the follow-up ANOVA confirmed the significant main effects of congruence, $F(1,49)=28.01, p<0.001$, partial $\eta^{2}=0.37$, and of cue type, $F(1,49)=13.57, p<0.01$, partial $\eta^{2}=0.22$. Performance was faster in the congruent $(M=490 \mathrm{~ms})$ than in the incongruent $(M=499 \mathrm{~ms})$ condition, 


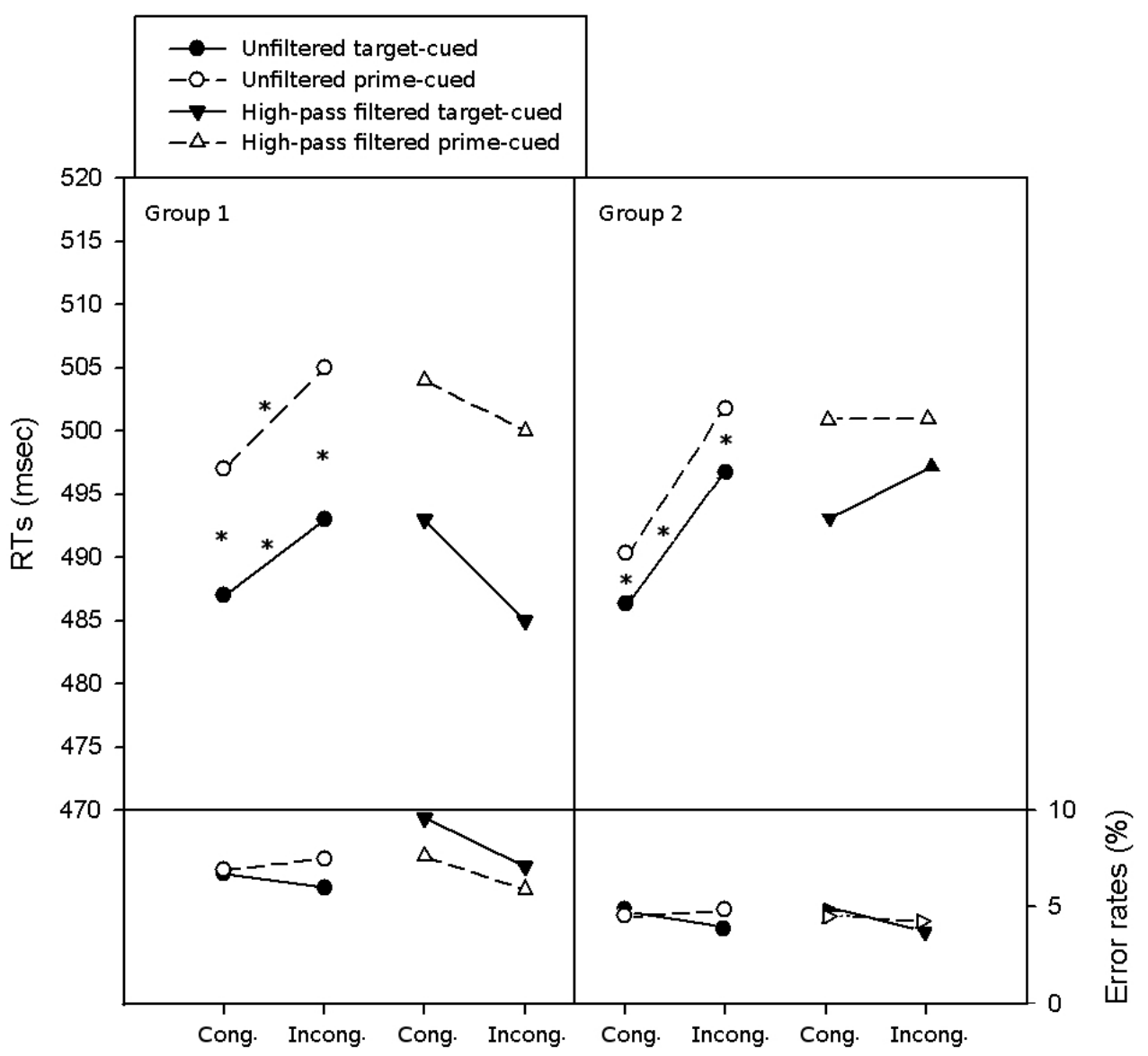

Figure 4. Mean Reaction Times (RTs) in milliseconds (upper panel) and error rates in percent (lower panel) on the y axis plotted as a function of congruence (congruent [Congrnt] vs. incongruent [Incongrnt]), prime type (unfiltered, high-pass filtered [HSF]), cue type (target-cued vs. prime-cued) and group (1: uncorrected HSF primes [left panel], 2: corrected for luminance and foreground-background contrast [right panel]) on the $\mathrm{x}$ axis, in Experiment 1 (circles = unfiltered conditions; triangles = high-pass filtered $[$ HSF] conditions; target-cued = solid lines and filled symbols; prime-cued $=$ dashed lines and unfilled symbols). Congruence effects can be seen in the unfiltered target-cued and prime-cued conditions of both groups 1 and 2, but not in the high-pass filtered conditions. Please note that the slight (although non-significant) positive tendency in group 2's high-pass filtered priming condition is also reflected in a comparatively higher congruence effect in the unfiltered priming condition of this group as compared to group 1. Additional details of the mean RTs, error rates, and prime visibilities are summarized in Table 1 . A star $\left(^{*}\right)$ shows that the effect of congruence or cue type was significant.

and in the target-cued $(M=491 \mathrm{~ms})$ than in the prime-cued $(M=499 \mathrm{~ms})$ condition. Also, in line with an attention-independence of the congruence effect, the two-way interaction of cue type and congruence was not significant, $F<1.00$. Attention-independence of the subliminal priming effect was predicted on the basis of classical theories of subliminal processing (cf. Posner \& Snyder, 1975). Attention-independence of at least the unfiltered primes' congruence effect is also in agreement with the refined position by Finkbeiner and Palermo (2009) according to which the masked priming effect is considered to be attention-independent.

In contrast, as expected, a similar follow-up ANOVA of the HSF primes showed no significant main effect of congruence, $F<1.00$, and only a significant main effect of the variable cue type, $F(1,49)=13.76, p<0.01$, partial $\eta^{2}=0.22$, with faster responses in the target-cued condition $(M=492 \mathrm{~ms})$ than in the prime-cued condition $(M=502 \mathrm{~ms})$. Unexpectedly on the basis of Finkbeiner and Palermo's (2009) hypothesis that cueing the HSF prime might gate its access to the parvo-cellular pathway even under masked conditions, there was also no significant interaction between cue type and congruence either, $F<1.00$.

With the HSF primes, there was also a significant two-way interaction of the variables congruence and group, $F(1,48)=4.70, p<0.04$, partial $\eta^{2}=0.09$. This interaction reflected that the congruence effect was $-5 \mathrm{~ms}$ in Group 1 but $2 \mathrm{~ms}$ in Group 2. This interaction might seem to argue for an influence of prime luminance and fo- 
Table 1. Mean reaction times (RTs), error rates, and results of prime visibility tests for the conditions of Experiment 1 . Mean RTs and error rates are compared in the congruent and incongruent conditions and the net congruence effect is calculated as the mean performance in the incongruent condition minus the mean performance in the congruent condition. Standard errors from the mean values are also provided. The results of $t$-tests against zero are given together with the mean $d$ ' values, averaged across congruent and incongruent conditions (Finkbeiner \& Palermo, 2009). The degrees of freedom for all $t$-tests of the mean $d$ 's were 24. Significance of a $t$-test was assumed if $p \leq 0.05$. The numbers in bold font indicate significant results. [Grp. = Group; Unfil. = Unfiltered; HSF = High-pass filtered; Congr. = Congruence; Cong. = Congruent; Incon. = Incongruent; Std. Err. = Standard Error; Sig. = Significance].

\begin{tabular}{|c|c|c|c|c|c|c|c|c|c|c|c|c|c|}
\hline \multicolumn{4}{|c|}{ Conditions } & \multicolumn{3}{|c|}{ Reaction Times (ms) } & \multicolumn{3}{|c|}{ Error rates (\%) } & \multicolumn{4}{|c|}{ Prime's visibility } \\
\hline Grp & Primes & $\begin{array}{c}\text { Cue-T }^{-T} \\
\text { ype }\end{array}$ & Congr. & $\begin{array}{c}\text { Mean } \\
\text { RTs }\end{array}$ & $\begin{array}{l}\text { Std. } \\
\text { Err. }\end{array}$ & $\begin{array}{l}\text { Net } \\
\text { Congr. }\end{array}$ & $\begin{array}{l}\text { Mean } \\
\text { Error } \\
\text { rates }\end{array}$ & $\begin{array}{l}\text { Std. } \\
\text { Err. }\end{array}$ & $\begin{array}{l}\text { Net } \\
\text { Congr. }\end{array}$ & $\begin{array}{l}\text { Mean } d \text { ' } \\
\text { (Cong. \& } \\
\text { Incon.) }\end{array}$ & $\begin{array}{l}\text { Std. } \\
\text { Err. }\end{array}$ & $\begin{array}{c}t \text {-test } \\
\text { against } 0\end{array}$ & $\begin{array}{c}\text { Sig. } \\
\text { (2-tailed) }\end{array}$ \\
\hline \multirow{8}{*}{1} & \multirow{4}{*}{ Unfil. } & \multirow{2}{*}{ Target } & Cong. & 487 & 9 & \multirow{2}{*}{6} & 6.7 & 0.9 & \multirow{2}{*}{-0.7} & \multirow{2}{*}{0.08} & \multirow{2}{*}{0.13} & \multirow{2}{*}{0.61} & \multirow{2}{*}{0.55} \\
\hline & & & Incon. & 493 & 10 & & 6.0 & 0.9 & & & & & \\
\hline & & \multirow{2}{*}{ Prime } & Cong. & 497 & 10 & \multirow{2}{*}{8} & 6.9 & 0.9 & \multirow{2}{*}{0.6} & \multirow{2}{*}{0.17} & \multirow{2}{*}{0.16} & \multirow{2}{*}{1.11} & \multirow{2}{*}{0.28} \\
\hline & & & Incon. & 505 & 11 & & 7.5 & 0.8 & & & & & \\
\hline & \multirow{4}{*}{ HSF } & \multirow{2}{*}{ Target } & Cong. & 493 & 9 & \multirow{2}{*}{-8} & 9.6 & 1.1 & \multirow{2}{*}{-2.5} & \multirow{2}{*}{0.06} & \multirow{2}{*}{0.13} & \multirow{2}{*}{0.47} & \multirow{2}{*}{0.64} \\
\hline & & & Incon. & 485 & 9 & & 7.1 & 0.9 & & & & & \\
\hline & & \multirow{2}{*}{ Prime } & Cong. & 504 & 11 & \multirow{2}{*}{-4} & 7.6 & 1.2 & \multirow{2}{*}{-1.7} & \multirow{2}{*}{0.13} & \multirow{2}{*}{0.12} & 106 & 030 \\
\hline & & & Incon. & 500 & 10 & & 5.9 & 0.9 & & & & 1.00 & 0.50 \\
\hline & & Trarøet & Cong. & 486 & 9 & 11 & 4.8 & 0.9 & $=0$ & - 10 & 011 & 097 & 030 \\
\hline & J I & Iarget & Incon. & 497 & 10 & 11 & 4.0 & 0.9 & -0.8 & -0.10 & 0.11 & $-0.8 \%$ & 0.39 \\
\hline & UाIII. & Drim & Cong. & 490 & 10 & 12 & 4.5 & 0.9 & 02 & (200 & 011 & 000 & 041 \\
\hline 7 & & Prrme & Incon. & 502 & 11 & 12 & 4.8 & 0.8 & 0.3 & 0.09 & 0.11 & 0.80 & 0.44 \\
\hline 2 & & & Cong. & 493 & 9 & 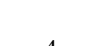 & 5 & 1.1 & & (2) & & & \\
\hline & & 1 arget & Incon. & 497 & 9 & 4 & 3.7 & 0.9 & -1.3 & -0.05 & 0.12 & -0.44 & 0.66 \\
\hline & ПDF & D. & Cong. & 501 & 11 & 0 & 4.6 & 1.2 & & & 01 & & דר \\
\hline & & Prrme & Incon. & 501 & 10 & 0 & 4.2 & 0.9 & -0.4 & -0.18 & 0.14 & -1 & 0.23 \\
\hline
\end{tabular}

reground-background contrast on the congruence effect. However, a similar although non-significant trend towards a lower congruence effect in Group 1 than 2 was found with the unfiltered primes, too. This common tendency for a between-group difference of the congruence effect in unfiltered as well as HSF filtered conditions was also reflected in the non-significant three-way interaction in the omnibus ANOVA. In addition, subsequent follow-up analyses of the HSF priming conditions, split up for Groups 1 and 2, showed that the congruence effect by the HSF primes was unreliable in both groups, Group $1, F(1,24)=3.00, p=0.09$, partial $\eta^{2}=0.11$, and Group 2, $F(1,24)=2.06, p=0.16$, partial $\eta^{2}=0.08$ (See Table 1 for the mean RTs and their stand errors).

\subsubsection{Error Rates}

The same omnibus ANOVA was also run separately on both percent and arcsine transformed error rates. Neither a significant main effect nor a significant interaction was found with either ANOVA, all $F s<1.00$. The mean error rates in each condition can be found in Figure 4 and Table 1.

\subsubsection{RT Distribution Analysis}

We were concerned that a residual mean congruence effect of the masked HSF primes could be found in only the fast responses (response priming) or only the slow responses. In other words, a more comprehensive test for residual congruence effects in the HSF conditions necessitated also looking at congruence effects as a function of the RT so as not to overlook congruence effects that were only lacking in the average RTs. As can be seen from Figure 5, this was not the case.

Formally, this assumption was corroborated in the following way. Correct RTs of each condition were sorted in an ascending order from fast to slow responses, and the resulting RT distribution was then split into 4 quartiles (bins). Taking the mean RT from each of these quartiles, we repeated the omnibus ANOVA as described 


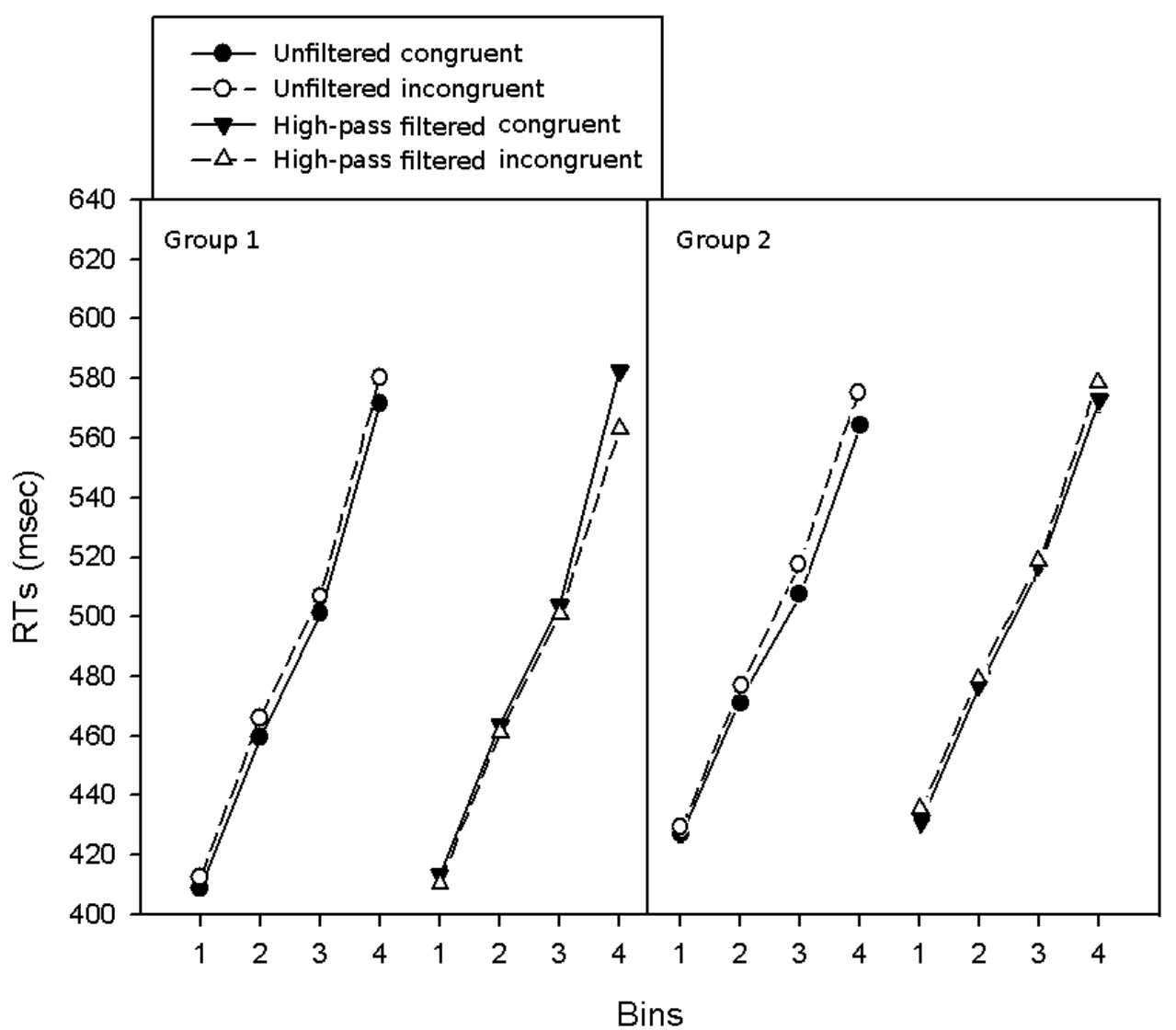

Figure 5. Mean Reaction Times (RTs) in milliseconds on the y axis plotted as a function of quartiles ( $1^{\text {st }}$ to $4^{\text {th }}$ bin of the RT distribution), prime type (unfiltered vs. high-pass filtered [HSF]), and congruence (congruent vs. incongruent) on the $\mathrm{x}$ axis, in Group 1 (left panel) and Group 2 (right panel) of Experiment 1 (congruent = solid lines and filled symbols; incongruent = dashed lines and unfilled symbols; circles = unfiltered; triangles = high-pass filtered). Congruence effects were almost equal in all quartiles of the RT distribution in the unfiltered priming conditions of both groups 1 and 2. Please note that the slight tendency towards a negative congruence effect in the high-pass filtered conditions of group 1 (left panel's triangular symbols) was more prominent in the slowest responses (i.e., the $4^{\text {th }}$ bin) but that this was not leading to a significant interaction of quartiles, congruence, and prime type.

above but with the additional variable quartile (or bin) of the RT distribution (1, 2, 3, 4). Besides the above mentioned significant effects and interactions of our variables, we did not find any other significant effect or interaction.

\subsubsection{Filler Trials}

Participants were able to discriminate the visible HSF faces used as targets in the filler trials of Group 2. Mean correct discrimination rate in the filler trials with HSF target faces $(M=72.5 \%, S E=3.0)$ was significantly different from chance level (50\%), $t(24)=7.40, p<0.01$. This means that our participants were able to successfully discriminate a face's gender on the basis of clearly visible (unmasked) HSF information alone and that a lack of inclusion of the HSF content in the participants' task sets does not provide an explanation of the absence of the congruence effect in HSF priming conditions.

\subsubsection{Prime Visibility}

Prime visibility was assessed in the prime-discrimination task using $d$ ' from signal detection theory (SDT). $d$ ' scores were obtained from direct calculation of hit rates and false alarm (FA) rates (cf. Green \& Swets, 1966). In the current study, 'male' primes were taken as 'signals' and 'female' primes as 'noise'. Here, $d$ ' is the z-transformed FA rate subtracted from the $z$-transformed hit rate. This index becomes zero in the case of chance performance and it can infinitely increase with ever increasing discrimination performance. One-sample $t$-tests in- 
dicated that the participants were not aware of the gender of the masked primes: $d$ 's were not significantly different from zero in any of the target-cued and prime-cued unfiltered priming and HSF priming conditions of both Groups 1 and 2, all $p$ s $>0.05$. The corresponding mean $d$ 's and the results of $t$-tests against zero can be found in Table 1. We additionally performed chi-squared tests for the target-cued unfiltered and filtered, and prime-cued unfiltered and filtered conditions. However, all of the results were non-significant, $p s>0.99$, thus replicating the above $d$ ' results.

\subsection{Discussion}

The results of Experiment 1 showed that filtering-out the low spatial frequencies from the masked peripheral face primes eliminated the congruence effect. This was in contrast to the unfiltered (broad-spectrum) face primes. These results are consistent with the hypothesis that the masked face priming effect arose from the magno-cellular pathway via the colliculi (Khalid et al., 2013). The results clearly indicated that the effective processing of the peripheral masked face primes depended on the type of spatial frequencies contained within them. When the face primes were unfiltered and thus contained both LSF and HSF bands, the faces were processed sufficiently to modulate the classification responses to the subsequent face targets. Also, the congruence effect by the unfiltered face primes was independent of shifting visual attention to the prime's location or to the target's location (see Finkbeiner \& Palermo, 2009). The lack of a congruence effect by the HSF primes under otherwise similar conditions showed that the briefly presented peripheral masked HSF primes that were not available to the magnocellular pathway were not processed so as to affect the subsequent target responses.

In addition, as can be seen in the participants' prime discrimination performance, our participants remained unaware of the masked primes' sexes. Taken together, the participants' unawareness of the face prime sexes and the dependency of their priming effect on LSF content neatly dovetail with prior assumptions that unaware face processing could be brought about by the retino-collicular projection (cf. Finkbeiner \& Palermo, 2009; Khalid et al., 2013; Tamietto \& De Gelder, 2010).

In Experiment 1, we also found that the foreground-background luminance and contrast equated HSF primes produced a trend towards a small positive congruence effect as compared to the uncorrected HSF primes. However, in a follow-up analysis in both groups the congruence effect of the masked HSF primes was non-significant. This minor influence of contrast on priming is well in line with known characteristics of peripheral vision during processing in the magno-cellular pathway (Croner \& Kaplan, 1995; Kaplan \& Shapley, 1986). The responses of the magno-cellular pathway rapidly increase with low contrasts and saturate at medium contrast values. In contrast, activity in the parvo-cellular neurons which are responsible for processing of visual content from the central (foveal) retinal regions increases with the contrast in visible (cf. Kaplan \& Shapley, 1986) and invisible stimuli (cf. Tapia \& Breitmeyer, 2011). The relative contrast-insensitivity of the congruence effect in the masked conditions of the present study would thus be also better in line with an origin of the congruence effect in the saturating range of the magno-cellular pathway's contrast sensitivity curve.

Somewhat surprisingly, Experiment 1 revealed that cueing the prime did not lead to a congruence effect with the masked HSF primes. This is in contrast to the presumed role of spatial attention in compensating for the loss of spatial detail in the visual periphery and, by extension, its role as a limiting factor in subliminal priming for non-face stimuli (cf. Finkbeiner \& Palermo, 2009). Maybe the spatial cue did never capture attention to the position of the prime. According to this explanation the facilitating effect of the spatial cue at target locations could have been due to a non-spatial effect, such as more temporal warning by a cue presented at the attended-to target location. This post-hoc explanation is, however, not so well in line with the boosting effect of prime cueing on priming effects in Experiment 2 of Finkbeiner and Palermo. It could thus also be that the present HSF primes were too weak to create a congruence effect even when attention was shifted to the location of the primes (Khalid et al., 2013).

\section{Experiment 2}

If collicular processing accounts for unaware processing in the face-sex discrimination task, we should find evidence for a congruence effect with masked LSF primes. By contrast, if any filtering decreases the amount of information conveyed by the primes and, thus, the masked congruence effect, we might find no congruence effects with masked LSF primes either. Thus, the purpose of Experiment 2 was to establish whether LSF faces provided sufficient information to produce a masked congruence effect on their own. 


\subsection{Method}

\subsubsection{Participants}

Thirty-eight fresh students (33 female) with a mean age of 23.0 years participated.

\subsubsection{Apparatus, Stimuli, and Procedure}

These were similar to Group 2 of Experiment 1, except that we used low-pass filtered face primes instead of high-pass filtered face primes (see Figure 2). To low-pass filter the images, a Gaussian function of 1 standard deviation and a cut-off frequency of 3cycles/image was applied to the original unfiltered images. The output was then cropped behind the oval layer. Also, as was the case for group 2 in Experiment 1, the filtered and unfiltered face primes were equated for luminance and foreground-background contrast, and 10\% filler trials, here with low-pass filtered target faces, were randomly interspersed.

\subsection{Results}

\subsubsection{Target-Discrimination Task}

Mean RTs can be found in Figure 6 and Table 2. As can be seen in Figure 6, there was a congruence effect for both the unfiltered (circular symbols) and the LSF (triangular symbols) priming conditions. This was corroborated by ANOVAs.

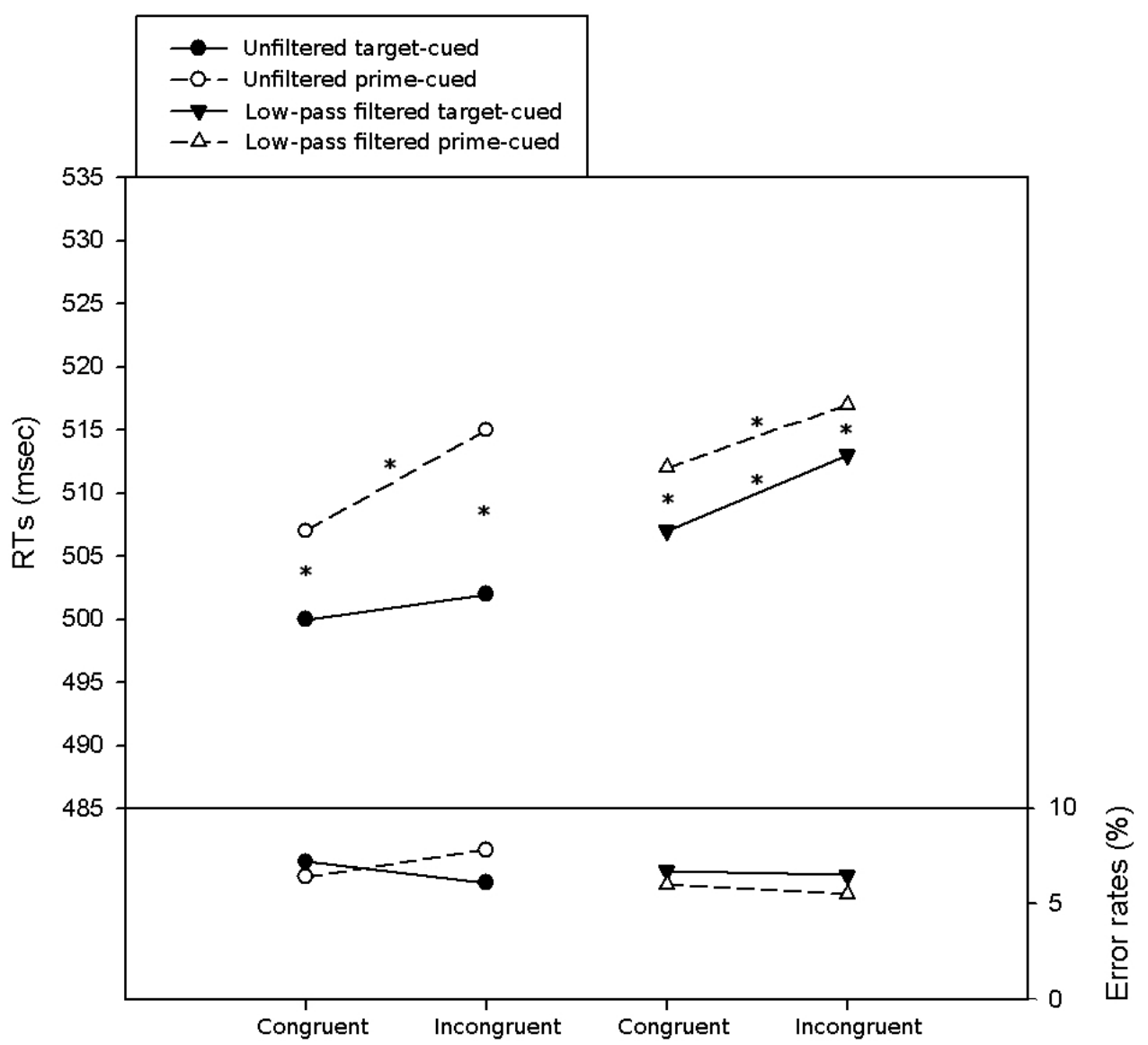

Figure 6. Mean Reaction Times (RTs) in milliseconds (upper panel) and error rates in percent (lower panel) on the y axis plotted as a function of congruence (congruent vs. incongruent), cue type (target-cued vs. prime-cued), and prime type (unfiltered vs. low-pass filtered [LSF]) in Experiment 2 on the $\mathrm{x}$ axis (unfiltered = circles; low-pass filtered = triangles; target-cued = solid lines and filled symbols; prime-cued = dashed lines and unfilled symbols). Congruence effects can be seen in the RTs of both the target-cued and prime-cued conditions of unfiltered and low-pass filtered (LSF) masked priming conditions. Details about the mean RTs and error rates in each of the conditions and results of the analysis of the prime's visibility are summarized in Table 2 . A star $(*)$ shows that the effect of congruence or cue type was significant. 
For the analysis of the correct mean RTs, $4.2 \%$ of the trials were eliminated for having RTs more than 2.5 SDs above or below the means. An omnibus repeated-measures ANOVA was run with the within-participant variables prime type (unfiltered primes, LSF primes), cue type (target-cued vs. prime-cued), and prime-target congruence (congruent vs. incongruent). There was a significant main effect of congruence: $F(1,37)=20.42, p$ $<0.001$, partial $\eta^{2}=0.37$. It was due to the participants' faster performance in the congruent ( $M=506 \mathrm{~ms}$ ) than in the incongruent $(M=512 \mathrm{~ms})$ condition. There was no significant interaction of congruence and prime type, $F<1.00, p>0.71$, partial $\eta^{2}=0.01$.The congruence effect of the LSF primes that is found here is in contrast to Experiment 1's HSF primes and shows that the LSF primes created a congruence effect of about the same size as the unfiltered face primes (See Table 2 for details).

Again, we found a significant main effect of the variable cue type, $F(1,37)=16.56, p<0.001$, partial $\eta^{2}=$ 0.31. Participants responded faster in the target-cued $(M=506 \mathrm{~ms})$ than in the prime-cued $(M=513 \mathrm{~ms})$ condition. Also, as before, in line with an attention-independence of the congruence effect, the factors cue type and prime type did not interact (all Fs $<1.00$ ).

\subsubsection{Error Rates}

The same omnibus ANOVA was also run separately on both percent and arcsine transformed error rates. No significant effect or interaction was found in either ANOVA, all $F \mathrm{~s}<1.00$. Mean error rates are depicted in Figure 6 and Table 2.

\subsubsection{Filler Trials}

Analogous to Experiment 1's Group 2, participants successfully discriminated the LSF target face images. Discrimination in the filler trials $(M=72.9 \%, S E=2.8$ ) was better than chance, $t(37)=8.07, p<0.001$.

\subsubsection{Distribution Analysis}

For the sake of completeness, we also ran a distribution analysis. As can be seen in Figure 7, an investigation of the congruence effects as a function of the response speed did not affect our conclusions. Formally, inclusion of the additional variable quartile of the RT distribution within the same omnibus ANOVA did not change the results. Besides the already reported main effects, we did not find any other significant effect or interaction.

\subsubsection{Prime Visibility}

Participants were not aware of the prime sex. This can be seen by the low mean $d$ 's and the non-significant $t$-tests in Table 2. d's were not significantly different from zero in any of the target-cued and prime-cued conditions, all $p s>0.05$. We additionally performed chi-squared tests for the unfiltered and filtered primes, separately

Table 2. Mean reaction times (RTs), error rates, and results of prime visibility tests for the conditions of Experiment 2. Mean RTs and error rates are compared in the congruent and incongruent conditions and the net congruence effect is calculated as the mean performance in the incongruent condition minus the mean performance in the congruent condition. Standard errors from the mean values are also provided. The results of $t$-tests against zero are given together with the mean $d$ ' values, averaged across congruent and incongruent conditions (Finkbeiner \& Palermo, 2009). The degrees of freedom for all $t$-tests of the mean $d$ 's were 37. The numbers in bold font indicate significant results. [Unfil = Unfiltered; LSF = Low-pass filtered; Congr. = Congruence; Cong. = Congruent; Incon. = Incongruent; Std. Err. = Standard Error; Sig. = Significance].

\begin{tabular}{|c|c|c|c|c|c|c|c|c|c|c|c|c|}
\hline \multicolumn{3}{|c|}{ Conditions } & \multicolumn{3}{|c|}{ Reaction Times (ms) } & \multicolumn{3}{|c|}{ Error rates (\%) } & \multicolumn{4}{|c|}{ Prime’s visibility } \\
\hline Primes & Cue-Type & Congr. & $\begin{array}{l}\text { Mean } \\
\text { RTs }\end{array}$ & $\begin{array}{l}\text { Std. } \\
\text { Err. }\end{array}$ & $\begin{array}{c}\text { Net } \\
\text { Congr. }\end{array}$ & $\begin{array}{c}\text { Mean } \\
\text { Error rates }\end{array}$ & $\begin{array}{l}\text { Std. } \\
\text { Err. }\end{array}$ & $\begin{array}{c}\text { Net } \\
\text { Congr. }\end{array}$ & $\begin{array}{c}\text { Mean } d^{\prime} \\
\text { (Cong. \&Incon.) }\end{array}$ & $\begin{array}{l}\text { Std. } \\
\text { Err. }\end{array}$ & $\begin{array}{c}\text { t-test } \\
\text { against } 0\end{array}$ & $\begin{array}{l}\text { Sig. } \\
\text { (2-tailed) }\end{array}$ \\
\hline \multirow{2}{*}{ Unfil. } & Target & $\begin{array}{l}\text { Cong. } \\
\text { Incon. }\end{array}$ & $\begin{array}{l}500 \\
502\end{array}$ & $\begin{array}{l}7 \\
7\end{array}$ & 2 & $\begin{array}{l}7.2 \\
6.1\end{array}$ & $\begin{array}{l}1.2 \\
1.0\end{array}$ & -1.1 & -0.1 & 0.23 & -0.44 & 0.67 \\
\hline & Prime & $\begin{array}{l}\text { Cong. } \\
\text { Incon. }\end{array}$ & $\begin{array}{l}507 \\
515\end{array}$ & $\begin{array}{l}8 \\
8\end{array}$ & 8 & $\begin{array}{l}6.4 \\
7.8\end{array}$ & $\begin{array}{l}0.8 \\
1.1\end{array}$ & 1.4 & 0.05 & 0.22 & 0.22 & 0.83 \\
\hline \multirow{2}{*}{ LSF } & Target & $\begin{array}{l}\text { Cong. } \\
\text { Incon. }\end{array}$ & $\begin{array}{l}507 \\
513\end{array}$ & $\begin{array}{l}6 \\
7\end{array}$ & 6 & $\begin{array}{l}6.7 \\
6.5\end{array}$ & $\begin{array}{l}0.7 \\
0.7\end{array}$ & -0.2 & -0.11 & 0.18 & -0.61 & 0.55 \\
\hline & Prime & $\begin{array}{l}\text { Cong. } \\
\text { Incon. }\end{array}$ & $\begin{array}{l}512 \\
517\end{array}$ & $\begin{array}{l}7 \\
8\end{array}$ & 5 & $\begin{array}{l}6.0 \\
5.5\end{array}$ & $\begin{array}{l}0.8 \\
0.7\end{array}$ & -0.5 & -0.37 & 0.19 & -1.9 & 0.07 \\
\hline
\end{tabular}




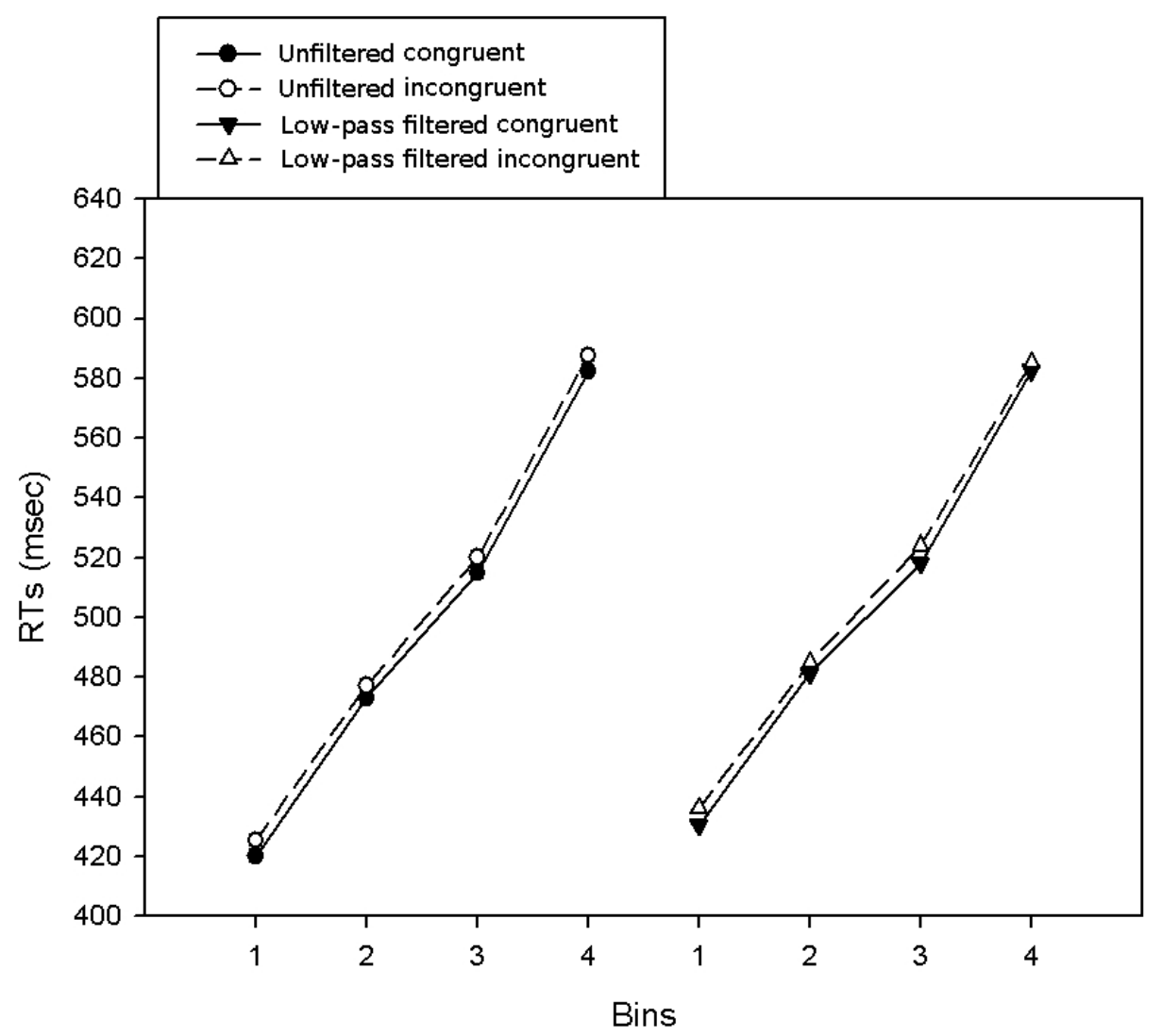

Figure 7. Mean Reaction Times (RTs) in milliseconds on the y axis plotted as a function of the quartile of the RT distribution ( $1^{\text {st }}$ to $4^{\text {th }}$ bin), prime type (unfiltered vs. low-pass filtered [LSF]), and congruence (congruent vs. incongruent) in Experiment 2 on the $\mathrm{x}$ axis (congruent = solid lines and filled symbols; incongruent $=$ dashed lines and unfilled symbols; circles = unfiltered priming condition; triangles = low-pass filtered priming condition). Congruence effects are of almost equal size in all quartiles of the RT distribution as can be seen in both unfiltered priming and low-pass filtered priming conditions.

for prime-cued and target-cued conditions. However, all of the results were non-significant, $p s>0.99$, thus replicating the above $d^{\prime}$ results.

\subsection{Discussion}

The results of Experiment 2 revealed that both masked unfiltered and masked LSF face primes produce a congruence effect. This finding lends support for the hypothesis that the retino-collicular projection drives the masked face priming effect because this projection is a branch of the magno-cellular pathway and the magno-cellular pathway is sensitive to the visual LSF signal (Johnson, 2005; Palermo \& Rhodes, 2007). In addition, the results showed that the congruence effect was the same for unfiltered and LSF primes, suggesting that the awareness-independent congruence effect could be even carried exclusively by the LSF content of the masked face primes. Finally, again the masked faces could not be seen by the participants and the processing of the masked primes was independent of the shifting of visual attention to either the target or to the prime.

\section{Experiment 3}

From the results of Experiment 1 it is clear that masked HSF primes do not lead to priming effects. However, participants' successful performance on high-pass filtered targets that were included on the filler trials in Experiment 1's group 2 indicated that critical sex information is carried by HSF content. This raises the interesting possibility that the successful use of sex information in HSF images is mediated by visual awareness (cf. De Gardelle \& Kouider, 2010). We tested this possible qualitative difference between unaware and aware processing in Experiment 3 by comparing the priming effects produced by HSF primes that were either masked or visible. 


\subsection{Method}

\subsubsection{Participants}

Twenty-eight fresh students (18 female) with a mean age of 21.5 years participated.

\subsubsection{Apparatus, Stimuli, and Procedure}

Because the contrast manipulation of Experiment 1 was without effect, we used conditions similar to that of group 1 in Experiment 1, with the exception that this experiment included a block of unmasked face primes. The trials with masked and unmasked primes were identical except that the masks (checkerboards and composites of scrambled faces) were replaced with blank screens (of black background color) to create the unmasked trials. Additionally, $10 \%$ filler trials with HSF face targets were included in both masked and unmasked conditions. Data from the filler trials were, however, again excluded from RT analysis and only analyzed separately to test for correct HSF target discrimination.

\subsection{Results}

\subsubsection{Target-Discrimination Task}

Mean RTs are shown in Figure 8 and Table 3.

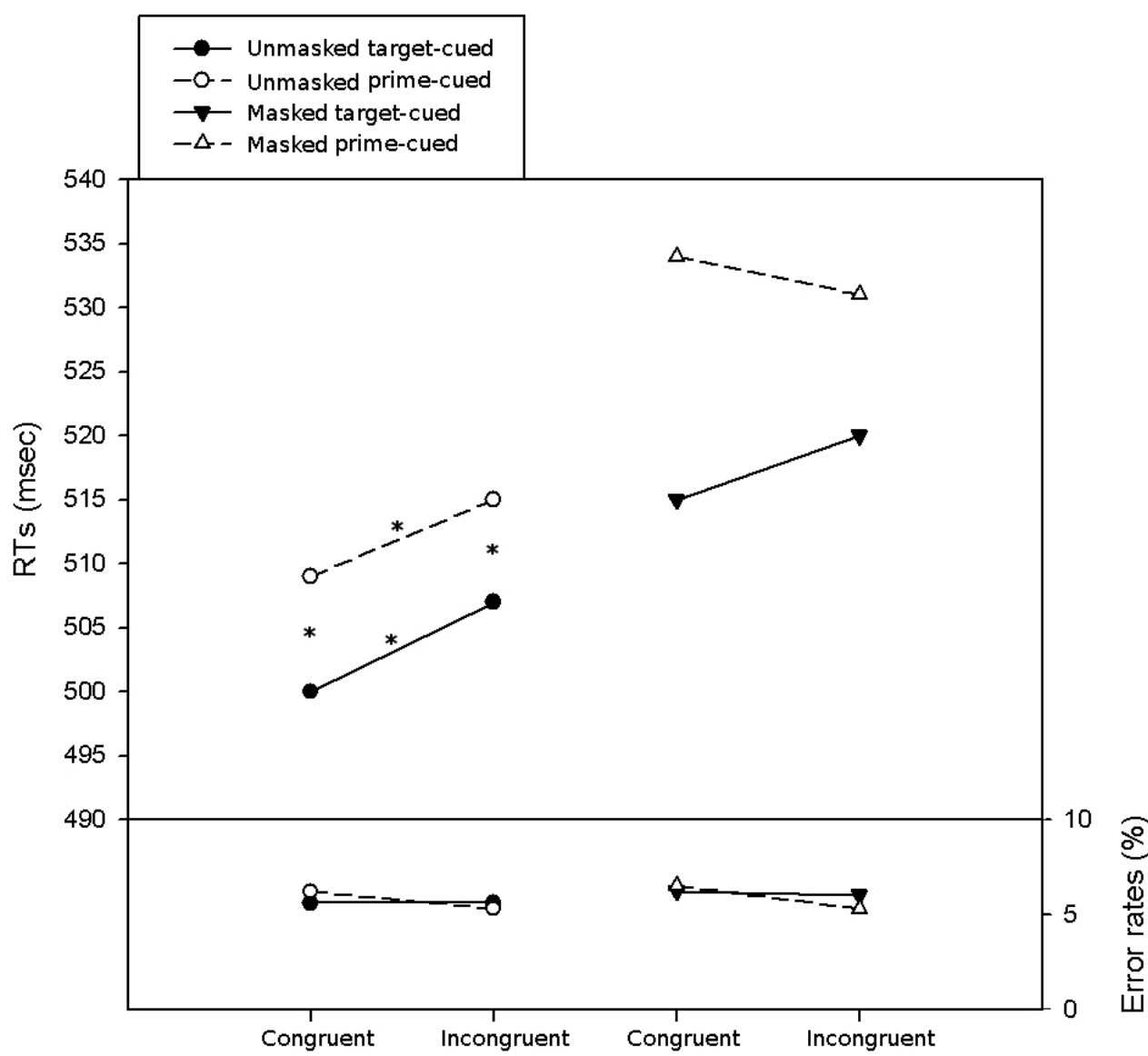

Figure 8. Mean Reaction Times (RTs) in milliseconds (upper panel) and error rates in percent (lower panel) on the y axis plotted as a function of the level of congruence (congruent vs. incongruent), cue-type (target-cued vs. prime-cued) and type of primes (unmasked high-pass filtered [HSF] vs. masked high-pass filtered [HSF]) in Experiment 3 on the $\mathrm{x}$ axis, (unmasked high-pass filtered = circles; masked high-pass filtered = triangles; target-cued condition = solid lines and filled symbols; prime-cued condition = dashed lines and unfilled symbols). Congruence effects can be observed only in the RTs of the unmasked high-pass filtered target-cued and prime-cued priming condition. The details about the mean RTs and error rates in each condition, and the results of the prime's visibility tests are summarized in Table 3. A star $(*)$ shows that the effect of congruence or cue type was significant. 
Table 3. Mean reaction times (RTs), error rates, and results of prime visibility tests for the conditions of Experiment 3. Mean RTs and error rates are compared in the congruent and incongruent conditions and the net congruence effect is calculated as the mean performance in the incongruent condition minus the mean performance in the congruent condition. Standard errors from the mean values are also provided. The results of $t$-tests against zero are given together with the mean $d$ ' values, averaged across congruent and incongruent conditions (Finkbeiner \& Palermo, 2009). The degrees of freedom for all $t$-tests of the mean $d$ 's were 27. The numbers in bold font indicate significant results. [HSF = High-pass filtered; Congr. = Congruence; Cong. = Congruent; Incon. = Incongruent; Std. Err. = Standard Error; Sig. = Significance].

\begin{tabular}{|c|c|c|c|c|c|c|c|c|c|c|c|c|}
\hline \multicolumn{3}{|c|}{ Conditions } & \multicolumn{3}{|c|}{ Reaction Times (ms) } & \multicolumn{3}{|c|}{ Error rates (\%) } & \multicolumn{4}{|c|}{ Prime's visibility } \\
\hline $\begin{array}{c}\text { Primes } \\
\text { HSF }\end{array}$ & Cue-Туре & Congr. & $\begin{array}{l}\text { Mean } \\
\text { RTs }\end{array}$ & $\begin{array}{l}\text { Std. } \\
\text { Err. }\end{array}$ & $\begin{array}{l}\text { Net } \\
\text { Congr. }\end{array}$ & $\begin{array}{l}\text { Mean Error } \\
\text { rates }\end{array}$ & $\begin{array}{l}\text { Std. } \\
\text { Err. }\end{array}$ & $\begin{array}{l}\text { Net } \\
\text { Congr. }\end{array}$ & $\begin{array}{l}\text { Mean d' } \\
\text { (Cong. } \\
\text { \&Incon.) }\end{array}$ & $\begin{array}{l}\text { Std. } \\
\text { Err. }\end{array}$ & $\begin{array}{c}t \text {-test } \\
\text { against } 0\end{array}$ & $\begin{array}{c}\text { sig. } \\
\text { (2-tailed) }\end{array}$ \\
\hline \multirow{4}{*}{ Unmask } & \multirow{2}{*}{ Target } & Cong. & 500 & 9 & \multirow{2}{*}{7} & 5.6 & 0.9 & \multirow{2}{*}{-0.1} & \multirow{2}{*}{0.33} & \multirow{2}{*}{0.12} & \multirow{2}{*}{2.82} & \multirow{2}{*}{0.01} \\
\hline & & Incon. & 507 & 10 & & 5.6 & 0.8 & & & & & \\
\hline & \multirow{2}{*}{ Prime } & Cong. & 509 & 10 & \multirow{2}{*}{6} & 6.2 & 0.9 & \multirow{2}{*}{-0.1} & \multirow{2}{*}{0.48} & \multirow{2}{*}{0.12} & \multirow{2}{*}{3.89} & \multirow{2}{*}{$\mathbf{0 . 0 0}$} \\
\hline & & Incon. & 515 & 9 & & 5.3 & 0.8 & & & & & \\
\hline \multirow{4}{*}{ Masked } & \multirow{2}{*}{ Target } & Cong. & 515 & 8 & \multirow{2}{*}{5} & 6.2 & 1.3 & \multirow{2}{*}{-0.4} & \multirow{2}{*}{-0.04} & \multirow{2}{*}{0.07} & \multirow{2}{*}{-0.64} & \multirow{2}{*}{0.53} \\
\hline & & Incon. & 520 & 9 & & 6.0 & 0.9 & & & & & \\
\hline & \multirow{2}{*}{ Prime } & Cong. & 534 & 9 & \multirow{2}{*}{-3} & 6.5 & 1.3 & \multirow{2}{*}{-0.3} & \multirow{2}{*}{-0.02} & \multirow{2}{*}{0.08} & \multirow{2}{*}{-0.30} & \multirow{2}{*}{0.77} \\
\hline & & Incon. & 531 & 9 & & 5.3 & 1.0 & & & & & \\
\hline
\end{tabular}

As can be seen from Figure 8, visible (unmasked) HSF primes (circular symbols) created a congruence effect, but invisible (masked) HSF primes (triangular symbols) failed to elicit a congruence effect. This conclusion was supported by our analysis.

Out of all correct responses, 3.7\% were excluded based on the same outlier criterion as was used in Experiments 1 and 2. The omnibus repeated-measures ANOVA of the correct mean RTs with the within-participant variables of masking (masked vs. unmasked), cue type (target-cued vs. prime-cued), and prime-target congruence (congruent vs. incongruent) led to a significant main effect of congruence, $F(1,27)=6.66, p<0.02$, partial $\eta^{2}=0.20$. This effect reflected that participants were significantly faster in congruent ( $\left.M=515 \mathrm{~ms}\right)$ than incongruent $(M=518 \mathrm{~ms})$ trials. Most importantly, a significant two-way interaction of the variables masking and congruence was observed, $F(1,27)=8.2, p<0.01$, partial $\eta^{2}=0.23$. This interaction reflected overall net congruence effects of $6 \mathrm{~ms}$ and $7 \mathrm{~ms}$ in the target-cued and prime-cued conditions respectively with the unmasked HSF primes which dropped respectively to $5 \mathrm{~ms}$ and $-3 \mathrm{~ms}$ with the masked HSF primes (see Table 3). This interaction was also further investigated by follow-up analyses (see below).

Also, we again found a significant main effect of cue type, $F(1,27)=50.47, p<0.001$, partial $\eta^{2}=0.65$. RTs were shorter in the target-cued condition $(M=511 \mathrm{~ms})$ than in the prime-cued condition $(M=522 \mathrm{~ms})$. In addition, the ANOVA led to a significant main effect of the variable masking, $F(1,27)=9.39, p<0.01$, partial $\eta^{2}=$ 0.26 , with faster performance in the unmasked condition $(M=508 \mathrm{~ms})$ than in the masked condition $(M=525$ $\mathrm{ms}$ ). This effect probably reflected the higher target discrimination demands in the masked conditions, in which the checkerboard (forward mask) and the compound face (backward mask) served as distractors and required task switching between relevant and irrelevant stimuli (or created competition with the target) as in rapid serial visual presentation streams (cf. Kawahara, Zuvic, Enns, \& Di Lollo, 2003; Keysers \& Perrett, 2002; Nieuwenstein, Chun, van der Lubbe, \& Hooge, 2005). Finally, we found a significant two-way interaction of the variables masking and cue-type, $F(1,27)=7.15, p<0.01$, partial $\eta^{2}=0.21$. The advantage of cueing the target as compared with cueing the prime was $15 \mathrm{~ms}$ in the masked condition but $8 \mathrm{~ms}$ in the unmasked condition. No other significant effect or interaction was found, all $F \mathrm{~s}<1.00$. Cueing the target was thus evidently more helpful in the more difficult (and noisier) masked conditions than in the less difficult (and less noisy) unmasked conditions, again corroborating the assumption that the sequential presentation of target and masking distractors created the target-discrimination difficulties (cf. Nieuwenstein et al., 2005).

A follow-up ANOVA of the masked priming condition with the within-participant variables cue type and congruence revealed again only a significant main effect of the variable cue type, $F(1,27)=46.17, p<0.001$, partial $\eta^{2}=0.63$, with faster responses in the target-cued $(M=518 \mathrm{~ms})$ than in the prime-cued $(M=533 \mathrm{~ms})$ condition. However, a similar follow-up ANOVA of the unmasked priming condition revealed a significant 
main effect of congruence, $F(1,27)=21.65, p<0.001$, partial $\eta^{2}=0.46$, in addition to the significant main effect of cue type, $F(1,27)=20.22, p<0.001$, partial $\eta^{2}=0.43$. With the unmasked HSF primes, participants performed faster in the congruent condition $(M=505 \mathrm{~ms})$ as compared with the incongruent condition $(M=511$ $\mathrm{ms})$. They also responded faster in the target-cued condition $(M=504 \mathrm{~ms})$ than in the prime-cued condition ( $M$ $=512 \mathrm{~ms}$ ). No other significant effect or interaction was found, all $F \mathrm{~s}<1.00$.

\subsubsection{Error Rates}

The same omnibus ANOVA was conducted separately with both percent and arcsine transformed error rates. No significant effect or interaction was found in any of the two ANOVAs, all $F \mathrm{~s}<1.00$, and all $p s>0.05$. Mean error rates can be seen in Figure 8 and Table 3.

\subsubsection{Filler Trials}

Participants successfully discriminated the HSF face targets' sex in the filler trials of both, the masked condition $(M=81.9 \%, S E=3.0), t(27)=10.56, p<0.001$, and the unmasked condition $(M=83.9 \%, S E=2.8), t(27)=$ $12.32, p<0.001$.

\subsubsection{Distribution Analysis}

Again, for the sake of completeness, we also ran a distribution analysis. As can be seen from Figure 9, the same

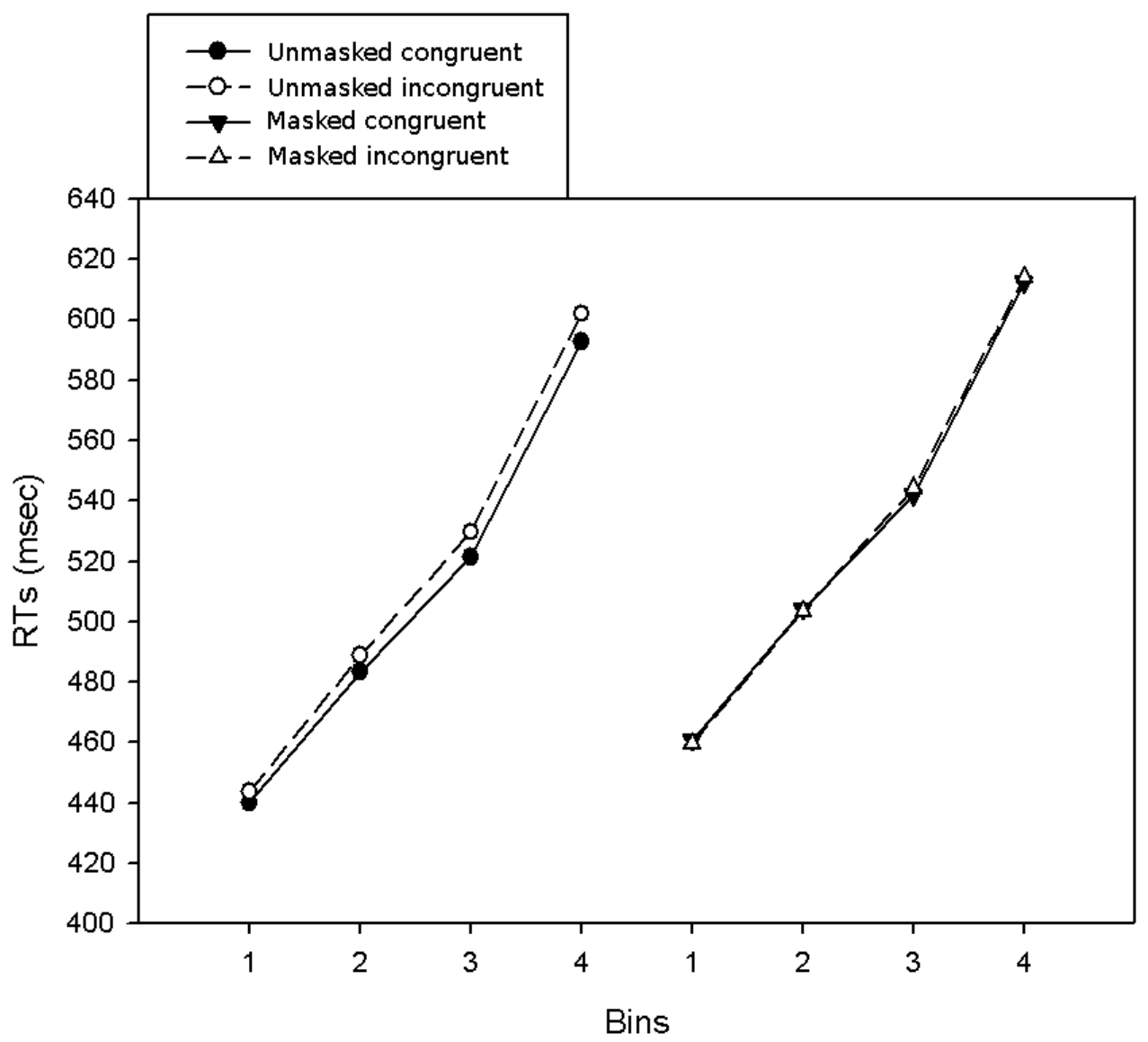

Figure 9. Mean Reaction Times (RTs) in milliseconds on the y axis plotted as a function of the quartiles of the RT distribution ( $1^{\text {st }}$ to $4^{\text {th }}$ bin of the RT distribution), prime type (unmasked high-pass filtered prime vs. masked high-pass filtered prime), and congruence (congruent vs. incongruent) on the $\mathrm{x}$ axis in Experiment 3 (congruent = solid lines and filled symbols; incongruent $=$ dashed lines and empty symbols; circles = unmasked high-pass filtered priming condition; triangles = masked high-pass filtered priming condition). Congruence effects of almost equal size can be seen in almost all quartiles for the unmasked high-pass filtered priming condition, but no congruence effect can be found in any of the quartiles with the masked high-pass filtered primes. 
conclusions were reached when we investigated the congruence effect as a function of the response speed. Like in Experiments 1 and 2, RTs were split into 4 quartiles (bins) and the same omnibus ANOVA was run with quartiles of the RT distribution $(1,2,3,4)$ as an additional within-participant variable. Apart from the above mentioned effects and interactions of our variables, we again did not find any other significant effect or interaction, all Fs $<1.00$.

\subsubsection{Prime Visibility}

Our participants were aware of the sex of the unmasked primes but not of that of the masked primes. This can be seen in Table 3. d's were calculated as in Experiments 1 and 2. Results showed that mean $d$ 's were not significantly different from zero in the masked target-cued condition $(M=-0.04)$, and the masked prime-cued condition $(M=-0.02)$, both $t s<1.00$. In contrast, mean $d$ 's were significantly different from zero in the unmasked target-cued condition $(M=0.33), t(27)=2.82, p<0.01$, as well as in the unmasked prime-cued condition $(M=$ $0.48), t(27)=3.89, p<0.01$. We additionally performed chi-square tests. The results were non-significant in the masked target-cued and prime cued conditions, $p s>0.99$, and were significant in the unmasked target-cued condition, $\mathrm{X}^{2}(1, N=27)=4.81, p<0.03$, and the unmasked prime-cued condition, $\mathrm{X}^{2}(1, N=27)=4.50, p<$ 0.04 , thus replicating the above $d$ ' results.

\subsection{Discussion}

The results of Experiment 3 confirmed that clearly visible (unmasked) HSF face primes led to a congruence effect. Also, the awareness of the participants for the sex of the unmasked HSF primes was tested and confirmed. Together, the congruence effect in the target discrimination task and the affirmed visibility of the unmasked HSF primes confirmed that HSF prime faces that are processed in an aware mode can contribute to face sex discrimination. Also, just as in Experiment 1 but this time in a within-subjects design, we again failed to find a congruence effect with masked HSF face primes. These face primes were not seen by our participants. Comparing the results from the aware and the unaware priming conditions, the different congruence effects are suggestive of a qualitative difference between aware and unaware sex classification processes when the critical information is carried by the HSF band alone. Note how this is different from what has been observed in a famejudgment task (De Gardelle \& Kouider, 2010) as explained above.

\section{General Discussion}

In altogether three experiments, we tested for a qualitative difference between supra- and subliminal priming. Based on the assumption that unaware but not aware processing of face sexes depended on the retino-collicular projection of the magno-cellular pathway, we expected congruence effects with masked unfiltered face primes but not with masked HSF primes. This prediction was confirmed in our present Experiment 1. Based on the same assumption, we tested as a second prediction whether masked LSF content of the face primes was sufficient to create a congruence effect. This prediction was confirmed in Experiment 2 in which we additionally showed that unfiltered face primes and LSF face primes created the same amount of congruence effects. Finally, the awareness-dependence of HSF priming was demonstrated in Experiment 3 in which we showed that unmasked HSF primes created a congruence effect but masked HSF primes once again failed to do so. Jointly, the results were in line with a qualitative processing difference between subliminal and supraliminal priming, particularly when filtered faces are used. Also, in all of our prime-discrimination tasks, we were able to confirm that the sexes of our masked faces were not reliably discriminated by our participants. In other words, participants were truly unaware of the masked face primes' sexes. In contrast, participants were able to discriminate the sexes of the unmasked face primes in Experiment 3.

Our observations partly align with the observed frequency-dependence of masked face priming in a face-fame judgment task: de Gardelle and Kouider (2010) found that priming with masked LSF faces did not depend on visibility but was found to be of the same strength regardless of the prime-mask interval and, thus, regardless of prime visibility. This was different with masked HSF priming. The congruence effect of the HSF faces increased with increasing prime-mask interval and, thus, was modulated by visibility. However, in contrast to the present study, these authors found masked priming in unaware conditions even with masked HSF faces. One possible reason for this difference is that de Gardelle and Kouider used a fame-judgment task. It is possible that fame judgments strongly depended on HSF content. In contrast, in the current study, we used face-sex discrimination. 
It is relatively clear that a sex-discrimination could be brought about by LSF content alone (Deruelle \& Fagot, 2005; Khalid et al., 2013; Laeng et al., 2010). An alternative possible difference between the studies concerns the construction of the filtered face primes. For example, we used one and the same image for different filtered and unfiltered versions of primes, whereas de Gardelle and Kouider combined two filtered faces to create their primes.

Our results also agree with the midbrain hypothesis of unaware vision (Johnson, 2005; Weiskrantz, 1986). For example, 'blindsight'-the residual visual processing capabilities in the visual field areas that are affected by scotoma after lesions to V1-have been attributed to the magno-cellular projection branching off into the midbrain's SC and bypassing relay via V1 (Weiskrantz, 1986; Weiskrantz et al., 1974). Even more to the point, certain facets of face processing, such as emotion discrimination, seem to be spared during blindsight, too (de Gelder, Vroomen, Weiskrantz, \& Pourtois, 1999; Pegna, Katheb, Lazeyras, \& Seghier, 2005; Tamietto \& De Gelder, 2010; see also Jolij \& Lamme, 2005). This does not mean that all awareness-independent LSF-based effects would be brought about by the midbrain projection and even less that face processing would rely on the midbrain projection alone. Some awareness-independent LSF-based effects seem to be brought about by cortical areas (Bar, 2003), and many well-understood face processing areas are located in the visual cortex (Kanwisher \&Yovel, 2009; McCarthy, Puce, Gore, \& Allison, 1997). Correspondingly, unaware face processing also occurs at cortical levels (Kouider et al., 2010), and subcortical processing of unaware face processing could be restricted to facial features of high adaptive value, such as sex discrimination and emotion recognition (cf. Kiss \& Eimer, 2008; Morris, Öhman, \& Dolan, 1999; Öhman, 2002).

The present results agree less well with recent findings that unaware vision could also draw on processing in the parvo-cellular pathway (Tapia \& Breitmeyer, 2011). Tapia and Breitmeyer used arrows as masked primes and targets (Vorberg et al., 2003). These authors found that the contrast-dependent variation of masked arrow priming was in line with predictions based on known contrast-sensitivity functions of the parvo-cellular pathway. Of course, the arrow discrimination task probably also required using the HSF band of the visual image rather than the LSF band. Whereas several results suggest that sex discrimination or emotion discrimination of faces can be brought about by LSF bands (Deruelle \& Fagot, 2005; Laeng et al., 2010; Vuilleumier, Armony, Driver, \& Dolan, 2003), HSF bands are critical for discriminating fine spatial detail as in shape perception, including the processing of (the identities of) faces (Vuilleumier et al., 2003) which is required in the fame-judgment task of Gardelle and Kouider (2010), too.

To account for all findings one therefore has to assume that participants have flexible control over the visual frequency band that is used to solve a visual discrimination task, and that an actually pertaining task set directed to selectively use the relevant visual features can be successfully applied to unconscious stimuli, too. Both these requirements have been affirmed to hold true. Humans have flexible control over the frequency band that they use in a visual discrimination task (Goffaux, Jemel, Jacques, Rossion, \& Schyns, 2003; Schyns, 1998; Schyns \& Oliva, 1999), and flexibly selected task sets can be applied to masked visual input, too (cf. Ansorge \& Neumann, 2005; Ansorge et al., 2014; Kunde, Kiesel, \& Hoffmann, 2003).

Before concluding, we also need to discuss two caveats of our study, one procedural and the other one concerning the findings. On a procedural level, besides the frequency band differences, there were visual characteristics by which filtered HSF and filtered LSF primes differed from one another. For example, the HSF images had a lower internal contrast and spectral power than the LSF images. In light of related findings, however, this difference does not seem to be critical. The absence of masked HSF priming and the presence of masked LSF priming can also be found where compensatory measures are taken to equate HSF and LSF images in terms of their respective internal image contrast and spectral power (Khalid et al., 2013). Of course, equating LSF and HSF primes for their respective contrast and spectral powers as was done by Khalid et al. also creates problems, such as increased noise levels of artifactual image content in the adjusted face images. These noise artifacts were not a problem with the unadjusted images that were used in the present study. The results of the present study and the one by Khalid et al. (2013) should be seen as complementary support for our conclusion.

Another topic of discussion of the present study concerns the lack of interaction between attention and priming in the masked HSF conditions that we found. Although this lack of interaction was predicted for the masked LSF primes, we were somewhat surprised to find no interaction between spatial cueing and priming for the HSF primes. In terms of the LSF faces, our observations were in line with Finkbeiner and Palermo's (2009) findings and their suggestion that LSF information can be utilized in an awareness-independent and an attention-independent manner (see also Posner \& Snyder, 1975). However, awareness-independent processing of HSF infor- 
mation should have been possible where attention was directed to the primes: Finkbeiner and Palermo argued that the sometimes observed attention-dependence of masked priming (Kiefer \& Brendel, 2006; Naccache, Blandin, \& Dehaene, 2002) would be typical of situations where unaware processing draws on HSF bands.

Yet, this was not found in the present study. Possibly, the current study's masked HSF primes were simply too weak to create an awareness-independent congruence effect in the attended conditions [but to note this finding has also been replicated with the adjusted HSF primes of Khalid et al. (2013)]. Alternatively, the cues might not have captured attention to the primes at all. It could be that the general advantage of target-cued as compared to prime-cued trials was due to a better or an exclusive non-spatial warning effect of the cues for the onset of the targets. This warning effect might have been stronger in or restricted to the target-cued conditions because only with a cue at the target position the cue was presented at the focus of attention. This post-hoc explanation is, however, not so well in line with prior findings by Finkbeiner and Palermo (2009) who found that cueing of the prime increased the priming effect.

\section{Conclusion}

In the present study, we tested and confirmed that unaware vision could sometimes selectively draw on LSF bands. This finding highlights that the retino-collicular projection could be one major origin of unaware face processing. In contrast, with clearly visible primes, no such limitation was found. With visible primes, even HSF primes created a congruence effect confirming that the parvo-cellular projection contributed to aware face processing. Together these findings support the view of a qualitative difference between aware and unaware modes of sex-face processing, and future studies should be aimed at testing similar discrepancies for other features, such as aware versus unaware processing of different emotional expressions.

\section{Acknowledgments}

Supported by, Deutsche Forschungsgemeinschaft Grant KH 341/1-1 to Shah Khalid, and an Australian Research Fellowship to Matthew Finkbeiner from the Australian Research Council (DP0880806). We are thankful to Bettina Füxl, Cathy Muller, Mascha Wisseler, Jörg-Alexander Kaiser, Julian Braehler, Christian Rammel, Bernadette Zecha, and Martin Kohl for help with the data collection.

Correspondence concerning this article should be addressed to Shah Khalid, Institute of Cognitive Science, University of Osnabrück, Osnabrück, Germany.

\section{References}

Ansorge, U., Horstmann, G., \& Worschech, F. (2010). Attentional Capture by Masked Color Singletons. Vision Research, 50, 2015-2027. http://dx.doi.org/10.1016/j.visres.2010.07.015

Ansorge, U., Kunde, W., \& Kiefer, M. (2014). Unconscious Vision and Executive Control: How Unconscious Processing and Conscious Action Control Interact. Consciousness and Cognition, 27, 268-287. http://dx.doi.org/10.1016/j.concog.2014.05.009

Ansorge, U., \& Neumann, O. (2005). Intentions Determine the Effects of Invisible Metacontrast-Masked Primes: Evidence for Top-Down Contingencies in a Peripheral Cueing Task. Journal of Experimental Psychology: Human Perception and Performance, 31, 762-777. http://dx.doi.org/10.1037/0096-1523.31.4.762

Bar, M. (2003). A Cortical Mechanism for Triggering Top-Down Facilitation in Visual Object Recognition. Journal of Cognitive Neuroscience, 15, 600-609. http://dx.doi.org/10.1162/089892903321662976

Breitmeyer, B. G., \& Ögmen, H. (2006). Visual Masking: Time Slices through Conscious and Unconscious Vision. Oxford: Oxford University Press. http://dx.doi.org/10.1093/acprof:oso/9780198530671.001.0001

Bullier, J. (2001). Integrated Model of Visual Processing. Brain Research Reviews, 36, 96-107. http://dx.doi.org/10.1016/S0165-0173(01)00085-6

Croner, L. J., \& Kaplan, E. (1995). Receptive Fields of P and M Ganglion Cells across the Primate Retina. Vision Research, 35, 7-24. http://dx.doi.org/10.1016/0042-6989(94)E0066-T

de Gardelle, V., \& Kouider, S. (2010). How Spatial Frequencies and Visual Awareness Interact during Face Processing. Psychological Science, 21, 58-66. http://dx.doi.org/10.1177/0956797609354064

de Gelder, B., Vroomen, J., Pourtois, G., \& Weiskrantz, L. (1999). Non-Conscious Recognition of Affect in the Absence of Striate Cortex. NeuroReport, 10, 3759-3763. http://dx.doi.org/10.1097/00001756-199912160-00007 
Dehaene, S., \& Naccache, L. (2001). Towards a Cognitive Neuroscience of Consciousness: Basic Evidence and a Workspace Framework. Cognition, 79, 1-37. http://dx.doi.org/10.1016/S0010-0277(00)00123-2

Deruelle, C., \& Fagot, J. (2005). Categorizing Facial Identities, Emotions, and Genders: Attention to High- and Low-Spatial Frequencies by Children and Adults. Journal of Experimental Child Psychology, 90, 172-184. http://dx.doi.org/10.1016/j.jecp.2004.09.001

Dimberg, U., Thunberg, M., \& Elmehed, K. (2000). Unconscious Facial Reactions to Emotional Facial Expressions. Psychological Science, 11, 86-89. http://dx.doi.org/10.1111/1467-9280.00221

Dixon, N. F. (1971). Subliminal Perception: The Nature of a Controversy. New York: McGraw-Hill.

Dolan, R. J., Fletcher, P., Morris, J., Kapur, N., Deakin, J. F. W., \& Frith, C. D. (1996). Neural Activation during Covert Processing of Positive Emotional Facial Expressions. NeuroImage, 4, 194-200. http://dx.doi.org/10.1006/nimg.1996.0070

Finkbeiner, M., \& Palermo, R. (2009). The Role of Spatial Attention in Nonconscious Processing: A Comparison of Face and Non-Face Stimuli. Psychological Science, 20, 42-51. http://dx.doi.org/10.1111/j.1467-9280.2008.02256.x

Forster, K. I., \& Davis, C. (1984). Repetition Priming and Frequency Attenuation in Lexical Access. Journal of Experimental Psychology: Learning, Memory, and Cognition, 10, 680-698. http://dx.doi.org/10.1037/0278-7393.10.4.680

Goffaux, V., Jemel, B., Jacques, C., Rossion, B., \& Schyns, P. G. (2003). ERP Evidence for Task Modulation on Face Perceptual Processing at Different Spatial Scales. Cognitive Science, 27, 313-325. http://dx.doi.org/10.1207/s15516709cog2702_8

Fuchs, I., Theeuwes, J., \& Ansorge, U. (2013). Exogenous Attentional Capture by Subliminal Abrupt-Onset Cues: Evidence from Contrast-Polarity Independent Cueing Effects. Journal of Experimental Psychology: Human Perception and Performance, 39, 974-988. http://dx.doi.org/10.1037/a0030419

Graham, D. J., \& Redies, C. (2010). Statistical Regularities in Art: Relations with Visual Coding and Perception. Vision Research, 50, 1503-1509. http://dx.doi.org/10.1016/j.visres.2010.05.002

Green, D. M., \& Swets, J. A. (1966). Signal Detection Theory and Psychophysics. New York: Wiley.

Greenwald, A. G., Draine, S. C., \& Abrams, R. L. (1996). Three Cognitive Markers of Unconscious Semantic Activation. Science, 273, 1699-1702. http://dx.doi.org/10.1126/science.273.5282.1699

Johnson, M. H. (2005). Subcortical Face Processing. Nature Reviews Neuroscience, 6, 766-774. http://dx.doi.org/10.1038/nrn1766

Jolij, J., \& Lamme, V. A. (2005). Repression of Unconscious Information by Conscious Processing: Evidence from Affective Blindsight Induced by Transcranial Magnetic Stimulation. Proceedings of the National Academy of Sciences, 102, 10747-10751. http://dx.doi.org/10.1073/pnas.0500834102

Kanwisher, N., \& Yovel, G. (2009). The Fusiform Face Area: A Cortical Region Specified for the Perception of Faces. Philosophical Transactions of the Royal Society B-Biological Sciences, 361, 2109-2128. http://dx.doi.org/10.1098/rstb.2006.1934

Kaplan, E., \& Shapley, R. M. (1986). The Primate Retina Contains two Types of Ganglion Cells, with Low and High Contrast Sensitivity. Proceedings of the National Academy of Sciences, 83, 2755-2757. http://dx.doi.org/10.1073/pnas.83.8.2755

Kawahara, J., Zuvic, S. M., Enns, J. T., \& Di Lollo, V. (2003). Task Switching Mediates the Attentional Blink Even without Backward Masking. Perception \& Psychophysics, 65, 339-351. http://dx.doi.org/10.3758/BF03194565

Keysers, C., \& Perrett, D. I. (2002). Visual Masking and RSVP Reveal Neural Competition. Trends in Cognitive Science, 6, 120-125. http://dx.doi.org/10.1016/S1364-6613(00)01852-0

Khalid, S., Finkbeiner, M., König, P., \& Ansorge, U. (2013). Subcortical Human Face Processing? Evidence from Masked Priming. Journal of Experimental Psychology: Human Perception and Performance, 39, 989-1002. http://dx.doi.org/10.1037/a0030867

Kiefer, M., \& Brendel, D. (2006). Attentional Modulation of Unconscious “Automatic” Processes: Evidence from EventRelated Potentials in a Masked Priming Paradigm. Journal of Cognitive Neuroscience, 18, 184-198. http://dx.doi.org/10.1162/jocn.2006.18.2.184

Kiss, M., \& Eimer, M. (2008). ERPs Reveal Subliminal Processing of Fearful Faces. Psychophysiology, 45, 318-326. http://dx.doi.org/10.1111/j.1469-8986.2007.00634.x

Kouider, S., Eger, E., Dolan, R. J., \& Henson, R. N. (2009). Activity in Face-Responsible Brain Regions by Invisible, Attended Faces: Evidence from Masked Priming. Cerebral Cortex, 19, 13-23. http://dx.doi.org/10.1093/cercor/bhn048

Kunde, W., Kiesel, A., \& Hoffmann, J. (2003). Conscious Control over the Content of Unconscious Cognition. Cognition, 88, 223-242. http://dx.doi.org/10.1016/S0010-0277(03)00023-4

Laeng, B., Profeti, I., Sæther, L., Adolfsdottir, S., Lundervold, A. J., Vangberg, T., Øvervoll, M., Johnsen, S. H., \& Waterloo, 
K. (2010). Invisible Expressions Evoke Core Impressions. Emotion, 10, 573-586. http://dx.doi.org/10.1037/a0018689

Lamme, V. A. F., \& Roelfsema, P. R. (2000). The Distinct Modes of Vision Offered by Feedforward and Recurrent Processing. Trends in Neurosciences, 23, 571-579. http://dx.doi.org/10.1016/S0166-2236(00)01657-X

Livingstone, M., \& Hubel, D. (1988). Segregation of Form, Color, Movement, and Depth: Anatomy, Physiology, and Perception. Science, 240, 740-749. http://dx.doi.org/10.1126/science.3283936

Lundqvist, D., Flykt, A., \& Öhman, A. (1998). The Karolinska Directed Emotional Faces-KDEF (CD ROM). Stockholm: Karolinska Institute, Department of Clinical Neuroscience, Psychology Section.

McCarthy, G., Puce, A., Gore, J. C., \& Allison, T. (1997). Face-Specific Processing in Human Fusiform Gyrus. Journal of Cognitive Neuroscience, 9, 605-610. http://dx.doi.org/10.1162/jocn.1997.9.5.605

Merigan, W. H., \& Maunsell, J. (1993). How Parallel Are the Primate Visual Pathways? Annual Review of Neuroscience, 16, 369-402. http://dx.doi.org/10.1146/annurev.ne.16.030193.002101

Morris, J. S., Öhman, A., \& Dolan, R. J. (1999). A Subcortical Pathway to the Right Amygdala Mediating “Unseen” Fear. Proceedings of the National Academy of Science, 96, 1680-1685. http://dx.doi.org/10.1073/pnas.96.4.1680

Mulckhuyse, M., \& Theeuwes, J. (2010). Unconscious Orienting to Exogenous Cues: A Review of the Literature. Acta Psychologica, 143, 199-209. http://dx.doi.org/10.1016/j.actpsy.2010.03.002

Naccache, L., Blandin, E., \& Dehaene, S. (2002). Unconscious Masked Priming Depends on Temporal Attention. Psychological Science, 13, 416-424. http://dx.doi.org/10.1111/1467-9280.00474

Nieuwenstein, M. R., Chun, M. M., van der Lubbe, R. H., \& Hooge, I. T. (2005). Delayed Attentional Engagement in the Attentional Blink. Journal of Experimental Psychology: Human Perception and Performance, 31, 1463-1475. http://dx.doi.org/10.1037/0096-1523.31.6.1463

Norris, D., \& Kinoshita, S. (2008). Perception as Evidence Accumulation and Bayesian Inference: Insights from Masked Priming. Journal of Experimental Psychology: General, 137, 434-455. http://dx.doi.org/10.1037/a0012799

Öhman, A. (2002). Automaticity and the Amygdala: Nonconscious Responses to Emotional Faces. Current Directions in Psychological Science, 11, 62-66. http://dx.doi.org/10.1111/1467-8721.00169

Olshausen, B. A., \& Field, D. J. (1996). Natural Image Statistics and Efficient Coding. Network: Computation in Neural Systems, 7, 333-339. http://dx.doi.org/10.1088/0954-898X_7_2_014

Palermo, R., \& Rhodes, G. (2007). Are You Always on My Mind? A Review of How Face Perception and Attention Interact. Neuropsychologia, 45, 75-92. http://dx.doi.org/10.1016/j.neuropsychologia.2006.04.025

Pegna, A. J., Khateb, A., Lazeyras, F., \& Seghier, M. L. (2005). Discriminating Emotional Faces without Visual Cortices Involves the Right Amygdala. Nature Neuroscience, 8, 24-25. http://dx.doi.org/10.1038/nn1364

Posner, M. I., \& Snyder, C. R. R. (1975). Attention and Cognitive Control. In R. L. Solso (Ed.), Information Processing and Cognition (pp. 55-85). Hillsdale, NJ: Erlbaum.

Purushothaman, G., Patel, S. S., Bedell, H. E., \& Ogmen, H. (1998). Moving Ahead through Differential Visual Latency. Nature, 396, 424-424. http://dx.doi.org/10.1038/24766

Reingold, E. M., \& Merikle, P. (1988). Using Direct and Indirect Measures to Study Perception without Awareness. Perception \& Psychophysics, 44, 563-575. http://dx.doi.org/10.3758/BF03207490

Schöberl, T., Fuchs, I., Theeuwes, J., \& Ansorge, U. (2015). Stimulus-Driven Attentional Capture by Subliminal Onset Cues. Attention, Perception, \& Psychophysics, 77, 737-748. http://dx.doi.org/10.3758/s13414-014-0802-4

Schyns, P. G. (1998). Diagnostic Recognition: Task Constraints, Object Information and Their Interactions. Cognition, 67, 147-179. http://dx.doi.org/10.1016/S0010-0277(98)00016-X

Schyns, P. G., \& Oliva, A. (1999). Dr. Angry and Mr. Smile: When Categorization Flexibly Modifies the Perception of Faces in Rapid Visual Presentations. Cognition, 69, 243-265. http://dx.doi.org/10.1016/S0010-0277(98)00069-9

Tailby, C., Cheong, S. K., Pietersen, A. N., Solomon, S. G., \& Martin, P. R. (2012). Colour and Pattern Selectivity of Receptive Fields in Superior Colliculus of Marmoset Monkeys. The Journal of Physiology, 590, 4061-4077.

http://dx.doi.org/10.1113/jphysiol.2012.230409

Tapia, E., \& Breitmeyer, B. G. (2011). Visual Consciousness Revisited Magnocellular and Parvocellular Contributions to Conscious and Nonconscious Vision. Psychological Science, 22, 934-942. http://dx.doi.org/10.1177/0956797611413471

Tamietto, M., \& de Gelder, B. (2010). Neural Basis of the Non-Conscious Perception of Emotional Signal. Nature Reviews Neuroscience, 11, 697-709. http://dx.doi.org/10.1038/nrn2889

Vath, N., \& Schmidt, T. (2007). Tracing Sequential Waves of Rapid Visuomotor Activation in Lateralized Readiness Potentials. Neuroscience, 145, 197-208. http://dx.doi.org/10.1016/j.neuroscience.2006.11.044

Vorberg, D., Mattler, U., Heinecke, A., Schmidt, T., \& Schwarzbach, J. (2003). Different Time Courses for Visual Percep- 
tion and Action Priming. Proceedings of the National Academy of Science, 100, 6275-6280. http://dx.doi.org/10.1073/pnas.0931489100

Vuilleumier, P., Armony, J. L., Driver, J., \& Dolan, R. J. (2003). Distinct Spatial Frequency Sensitivities for Processing Faces and Emotional Expressions. Nature Neuroscience, 6, 624-631. http://dx.doi.org/10.1038/nn1057

Weiskrantz, L. (1986). Blindsight: A Case Study and Implication. Oxford: Oxford University Press.

Weiskrantz, L., Warrington, E. K., Sanders, M. D., \& Marshall, J. (1974). Visual Capacity in the Hemianopic Field Following a Restricted Occipital Ablation. Brain, 97, 709-728. http://dx.doi.org/10.1093/brain/97.1.709 